\title{
Modified double-averaged Hamiltonian in hierarchical triple systems
}

\author{
Hanlun Lei ${ }^{\oplus},{ }^{1 \star}{\text { Christian } \text { Circi }^{2} \text { and Emiliano Ortore }}^{2}$ \\ ${ }^{1}$ School of Astronomy and Space Science, Nanjing University, Nanjing 210046, China \\ ${ }^{2}$ Department of Astronautical, Electrical and Energy Engineering, Sapienza University of Rome, Via Salaria, 851, I-00138 Rome, Italy
}

Accepted 2018 September 19. Received 2018 September 18; in original form 2018 July 25

\begin{abstract}
In this work, we introduce a modified double-averaging approach by considering the shortterm effects and formulate a more accurate double-averaged Hamiltonian (in comparison to the classical octupole-level Hamiltonian) for hierarchial triple systems. The Hamiltonian is expressed as a power series in the ratio of the semi-major axes of the inner and outer binaries. Both the Delaunay's elements and the classical orbit elements are adopted to describe the motion. To derive the secular Hamiltonian, the short-term oscillations in the Hamiltonian are averaged out by means of a double-averaging approach. In particular, during the average over the orbital period of the outer binary, the periodic corrections to the secular motion are taken into account. Based on the double-averaged Hamiltonian, we provide two versions of equations of secular motion, given in the form of canonic relations and Lagrange planetary equations. The resulting secular evolution equations can be utilized to reproduce the long-term behaviours for those physical systems where the perturbations coming from the disturbing bodies are relatively strong. To test the approach, we use the averaged Hamiltonian to predict the longterm motions of a planet in a stellar binary system and natural satellites in Sun-planet systems. Simulation results show that the modified Hamiltonian can reproduce secular behaviours with high accuracy. Additionally, the comparison of dynamical models truncated at different orders indicates that the secular Hamiltonian with inclusion of higher order terms has better accuracy in predicting long-term evolution.
\end{abstract}

Key words: celestial mechanics - methods: analytical - planets and satellites: dynamical evolution and stability.

\section{INTRODUCTION}

In a triple system, the stability condition requires that the three bodies constitute a circular, concentric and coplanar configuration or a hierarchical configuration (Naoz 2016). The hierarchical physical systems are common in the Universe, such as planet-satellite systems with the Sun as a distant perturber, planets in stellar binaries and stellar binaries perturbed by massive black holes.

Several decades ago, Allan \& Cook (1964) adopted the Milankovitch elements (the eccentricity vector and the normalized angular momentum vector) to describe the motion of probes, and developed the (secular) Lagrange planetary equations in the vectorial form by performing a double average for the disturbing function. In their studies, the third body is located in an elliptic and inclined orbit, and the disturbing function is truncated at the second order in the semi-major axial ratio $\alpha=a_{1} / a_{2}$ with $a_{1}$ and $a_{2}$ as the semi-major axes of the inner and outer binaries, respectively. Then, based on these secular vectorial equations, many works have been performed, e.g. Circi, Condoleo \& Ortore (2017) adopted the equations to determine families of solutions at constant mean orbit elements, and Condoleo et al. (2016) retrieved families of frozen orbits with equatorial perturbing bodies.

Also in the 1960s, Lidov (1962) adopted the classical orbit elements to describe the motion of satellites and investigated the secular behaviours around the Earth under the gravitational perturbations coming from the external bodies (the Moon and the Sun), and Kozai (1962) studied the secular influences of Jupiter's gravitational perturbations upon the highly inclined and eccentric asteroids in our Solar system. Both of these two landmark works were performed under the following assumptions: (a) the bodies of interest (the satellites and asteroids) are assumed as massless particles, (b) the perturbers move around the central body in circular orbits, leading to axisymmetric outer potentials, and (c) the disturbing functions are truncated at the second order (quadrupole-level approximation) in the semi-major axial ratio. Considering 
these approximations, they found that (a) the Kepler energy of each binary's orbit is conserved, leading to the fact that the semi-major axes of the inner and outer binaries keep stationary in the secular sense and (b) the angular momentum projected on the total angular momentum vector of the whole system ( $z$ axis), $H=\sqrt{1-e^{2}} \cos i$, is conserved, leading to coupled oscillations of the eccentricity (e) and inclination (i). Naoz (2016) called the assumptions adopted by Lidov (1962) and Kozai (1962) as the test particle quadrupole (TPQ) approximation. Under the TPQ setting, the system is integrable and the eccentricity of the test particle could be excited up to a high value due to the 'Kozai resonance' when $i>39.2^{\circ}$ (Thomas \& Morbidelli 1996). This mechanism of eccentricity excitation is often called the Kozai-Lidov (KL) mechanism.

In recent years, Naoz et al. (2011, 2013), Li et al. (2014a) and many other researchers have relaxed the TPQ limit to a more general case: (a) the distant perturbing body is in an eccentric orbit, (b) the three bodies in a triple system are massive, and (c) the disturbing function is relaxed to the next level of approximation in the semi-major axial ratio, i.e. at the octupole-order approximation. These extensions lead to a more generalized version of KL mechanism, called the eccentric Kozai-Lidov (EKL) mechanism (Naoz 2016), which has been widely used to describe the dynamical phenomena of astrophysical triple systems on time-scales much longer than the orbital periods.

In the EKL setting, the $z$-component of the inner or outer orbit's angular momentum is no longer conserved, and the secular evolution of the system is a result of exchanging angular momenta of the inner and outer orbits. As the system is chaotic (Li et al. 2014b), it is possible for both the inner or outer orbit to switch between prograde and retrograde with respect to the total angular momentum (Naoz et al. 2013, 2017). Under the octupole-level approximation, Naoz et al. (2011) studied the secular evolution of a triple system composed of an inner compact binary including a star and a Jupiter-like planet, perturbed by a distant Jupiter-mass planet (or brown dwarf). The secular perturbation coming from the perturber drives large oscillations of the eccentricity and inclination of the inner orbit. Sometimes, the inclination may even change its sign (orbit flipping). The flipping event is usually accompanied by an excursion to very high eccentricity, resulting in a very low altitude of periastron for the inner planet. This provides a possible mechanism of formation for retrograde 'hot Jupiters': the low altitude of the periastron during the secular evolution enhances planet-star tidal interactions that circularize the inner orbit, resulting in decoupling the planets and forming a retrograde hot Jupiter (Naoz et al. 2011).

To study the secular dynamics about a multibody system, it is required to formulate a suitable dynamical model governing the secular evolution. To this end, the Hamiltonian is usually expressed as a power series in the ratio of semi-major axes of the inner and outer orbits. Then, by means of a double-averaging technique, the short-term effects appearing in the Hamiltonian can be eliminated, yielding the equations of secular motion. As for this issue, Laskar \& Boué (2010) developed a general approach to formulate the expansion of secular perturbations of hierarchical triple systems in terms of Legendre polynomials at any order in the semi-major axial ratio. Under the assumption that the disturbing body's orbit is fixed, Carvalho et al. (2016) derived an explicit expression for the double-averaged disturbing function up to the fifth order in the semi-major axial ratio and used it to study the effects of the higher order terms in the potential function on the secular evolution. Will (2017) formulated an expansion of the equations of secular motion up to hexadecapole order and incorporated the relativistic effects in the hexadecapole-level model to analyse the flip behaviour of the disturbed body. Hamers \& Portegies (2016) presented a formalism and an algorithm to explicitly derive the double-averaged Hamiltonian (in vectorial form) for triple systems up to and including fifth order (dotriacontupole order) and formally to any order, and they generalized the model to study the secular dynamics of hierarchical multiple systems with more than three bodies. Actually, the double-averaged disturbing function (or secular Hamiltonian) truncated at the third order (octupole-level approximation) has been widely used in previous researches (Katz, Dong \& Malhotra 2011; Lithwick \& Naoz 2011; Naoz et al. 2011, 2013; Li et al. 2014a,b; Antognini 2015; Petrovich 2015).

Recently, Luo, Katz \& Dong (2016) showed that the periodic effects neglected by the classical double averaging can accumulate over long time-scales, which could produce significant errors in predicting the long-term behaviours (in history a similar phenomenon has been observed in the lunar evection problem). In particular, they found that the dynamical model formulated by the classical double averaging may fail in characterizing the long-term evolution when the perturbations coming from the disturbing bodies are relatively strong. Actually, a variety of astrophysical systems are related to this issue, such as stellar binaries orbited by massive black holes and satellites of planets perturbed by the Sun. To address this problem, they adopted a method similar to that presented in Ćuk \& Burns (2004) to derive corrected double-averaged equations by incorporating the effects of short-term oscillations into the double averaging. The disturbing function they obtained is truncated at the octupole level, and the periodic correction is considered at the quadrupole level. For this issue, it would be important to generalize the 'corrected double averaging' to a higher order (not limited to the octupole-level Hamiltonian and quadrupole-level correction), so as to extend the range of applications. This motivates us to carry out the current work.

In this study, we aim to develop a modified double-averaging technique and formulate a generalized expression of the secular Hamiltonian truncated at an arbitrary order. In this technique, the effects of short-term oscillations are incorporated into the double averaging. The modified double averaging is used to formulate a generalized form of the secular Hamiltonian. In particular, two versions of double-averaged systems are retrieved from the canonical relations and Lagrange planetary equations.

The structure of this paper is organized as follows. In Section 2, the Hamiltonian is expressed as a function of the orbit elements of the inner and outer binaries. In Section 3, the Hamiltonian is averaged over the orbital period of the inner binary, and the explicit expressions of the periodic oscillations are obtained. In Section 4, the final form of the secular Hamiltonian is retrieved by taking the periodic oscillations into consideration, and the equations of secular motion are provided. In Section 5, we apply the obtained dynamical model to a stellar system consisting of two massive stars and one massless planet and to three Sun-perturbed planet-satellite systems. Finally, Section 6 concludes this work. 


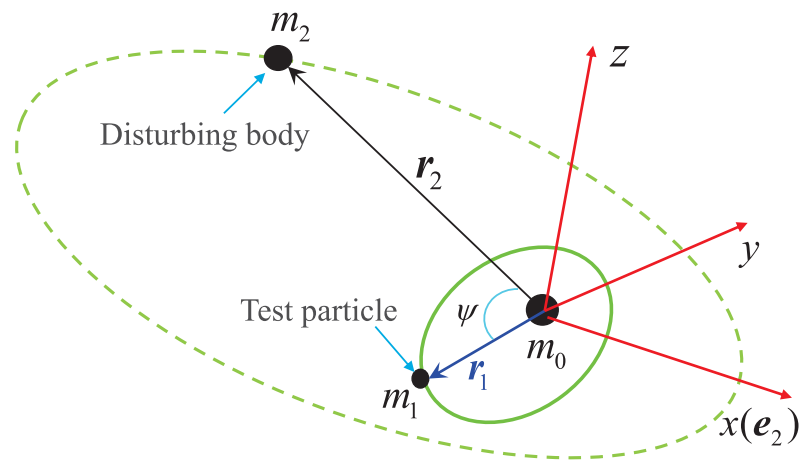

Figure 1. Relative geometry of three bodies in a hierarchial triple system (not to scale) and schematic diagram of the inertial coordinate system. The mass parameters of this triple system are $m_{0}$ for the central body, $m_{1}$ for the test particle and $m_{2}$ for the faraway disturbing body. The $x-y$ plane of the coordinate system coincides with the motion plane of the disturbing body, the $x$-axis is set in the direction of the eccentricity vector $\boldsymbol{e}_{2}$ and the $z$-axis is aligned along the angular momentum vector of the disturbing body's orbit. In the coordinate system $o-x y z$, vector $\boldsymbol{r}_{\mathbf{1}}$ points from $m_{0}$ to $m_{1}$, and vector $\boldsymbol{r}_{2}$ goes from $m_{0}$ to $m_{2}$. The relative angle between the position vectors $\boldsymbol{r}_{1}$ and $\boldsymbol{r}_{\mathbf{2}}$ is denoted by $\psi$.

\section{HAMILTONIAN FUNCTION}

The triple system considered in this study consists of a central body with mass $m_{0}$, a test particle with mass $m_{1}$ and a disturbing body with mass $m_{2}$. In the test particle limit, $m_{1}$ is negligible with respect to $m_{0}$ and $m_{2}$. Thus, the gravitational influence of the test particle upon the motions of the other two massive bodies can be neglected, leading to the consequence that the motion of the disturbing body around the central body is analytical (i.e. Kepler orbit). The dynamical model, describing the motion of the test particle, is often called the restricted three-body problem (RTBP), in which the motions around the collinear and triangular equilibrium points have been analytically studied in Lei \& Xu (2013, 2014), Lei et al. (2013) and Lei, Xu \& Circi (2018).

To describe the motions of the triple system, the following inertial coordinate system, denoted by $o-x y z$, is defined: the origin is located at the central body, the starting axis $(x)$ is aligned along the eccentricity vector of the disturbing body's orbit, the $z$-axis is parallel to its orbital angular momentum vector and the $y$-axis is determined to complete a right-handed coordinate system (see Fig. 1 for the detailed illustration). In the defined reference frame, Kepler motions can be characterized by means of the classical orbit elements. In particular, the orbital shape is determined by the semi-major axis $a_{j}$ and the eccentricity $e_{j}$, the orbital orientation is expressed by the inclination $i_{j}$, the longitude of the ascending node $\Omega_{j}$ and the argument of pericentre $\omega_{j}$, and the position of the body of interest is specified by the mean anomaly $M_{j}$ (or the eccentric anomaly $E_{j}$, or the true anomaly $f_{j}$ ), where $j=1$ is for the test particle and $j=2$ for the disturbing body. Unless otherwise specified, we use the subscript 1 to stand for the test particle and 2 for the disturbing body in the whole paper.

We are dealing with a hierarchical triple system, which can be treated as two isolated binaries: the inner binary consisting of the central body and the test particle, and the outer binary composed of the central body and the disturbing body. A hierarchical configuration requires that the semi-major axis of the test particle's orbit is much smaller than that of the disturbing body's orbit. Therefore, the ratio of the semi-major axes, $\alpha=a_{1} / a_{2}$, is a small parameter. According to Harrington (1969), the motion of the test particle can be described by the following Hamiltonian:

$\mathcal{H}=-\frac{\mu}{2 a_{1}}-\frac{\mathcal{G} m_{2}}{a_{2}} \sum_{n \geq 2}^{\infty} \alpha^{n}\left(\frac{r_{1}}{a_{1}}\right)^{n}\left(\frac{a_{2}}{r_{2}}\right)^{n+1} P_{n}(\cos \psi), \quad n \in \mathbb{N}$,

where $\mu=\mathcal{G} m_{0}$ with $\mathcal{G}$ as the gravitational constant, $r_{1}$ and $r_{2}$ are, respectively, the magnitudes of the position vectors $\boldsymbol{r}_{1}$ (for body 1) and $\boldsymbol{r}_{2}$ (for body 2), and $P_{n}\left({ }^{*}\right)$ is the Legendre polynomial with degree $n$. In addition, $\psi$ is the relative angle between $\boldsymbol{r}_{1}$ and $\boldsymbol{r}_{2}$, determined by

$\cos \psi=\frac{\boldsymbol{r}_{1}}{r_{1}} \cdot \frac{\boldsymbol{r}_{2}}{r_{2}}$

In equation (1), the first term in the right-hand side corresponds to the Kepler energy of the test particle, and the second term is often called the disturbing potential (or disturbing function), describing the mutual interaction between the orbits of the test particle and the disturbing body.

For convenience, we denote the disturbing function by

$\mathcal{R}=\frac{\mathcal{G} m_{2}}{a_{2}} \sum_{n \geq 2}^{\infty} \alpha^{n}\left(\frac{r_{1}}{a_{1}}\right)^{n}\left(\frac{a_{2}}{r_{2}}\right)^{n+1} P_{n}(\cos \psi)$,

where the Legendre polynomial term can be expanded as

$P_{n}(\cos \psi)=\sum_{k \geq 0}^{\left[\frac{n}{2}\right]} \frac{(-1)^{k}}{2^{n}} \frac{(2 n-2 k) !}{k !(n-k) !(n-2 k) !} \cos ^{n-2 k} \psi, \quad k \in \mathbb{N}$ 
with $\left[\frac{n}{2}\right]$ as the integer part of $\frac{n}{2}$. The same expansion can be found in Laskar \& Boué (2010). As a result, the Hamiltonian can be written as

$\mathcal{H}=-\frac{\mu}{2 a_{1}}-\mathcal{R}$.

In this study, we adopt the canonical variables known as Delaunay's elements, including three angles and three conjugate momenta, to describe the motion of the test particle. The three angles are $l, g$, and $h$, and their corresponding conjugate momenta are, respectively, denoted by $L, G$, and $H$. The Delaunay's elements are defined by means of the classical elements in the following form:

$\begin{array}{ll}L=\sqrt{\mu a_{1}}, & l=M_{1}, \\ G=L \sqrt{1-e_{1}^{2}}, & g=\omega_{1}, \\ H=G \cos i_{1}, & h=\Omega_{1},\end{array}$

where $L$ is related to the Kepler energy $(K)$ of the test particle by $K=-\frac{\mu^{2}}{2 L^{2}}, G$ is the magnitude of the angular momentum vector, and $H$ corresponds to the angular momentum along the $z$ direction. Concerning the test particle's motion, a prograde orbit is defined by $i_{1}<90^{\circ}$ $(H>0)$, and a retrograde orbit by $i_{1}>90^{\circ}(H<0)$.

Using Delaunay's elements, the Hamiltonian becomes

$\mathcal{H}=-\frac{\mu^{2}}{2 L^{2}}-\mathcal{R}$,

and the equations of motion are given by the following canonical relations:

$\frac{\mathrm{d} l}{\mathrm{~d} t}=\frac{\partial \mathcal{H}}{\partial L}, \quad \frac{\mathrm{d} L}{\mathrm{~d} t}=-\frac{\partial \mathcal{H}}{\partial l}$,

$\frac{\mathrm{d} g}{\mathrm{~d} t}=\frac{\partial \mathcal{H}}{\partial G}, \quad \frac{\mathrm{d} G}{\mathrm{~d} t}=-\frac{\partial \mathcal{H}}{\partial g}$,

$\frac{\mathrm{d} h}{\mathrm{~d} t}=\frac{\partial \mathcal{H}}{\partial H}, \quad \frac{\mathrm{d} H}{\mathrm{~d} t}=-\frac{\partial \mathcal{H}}{\partial h}$.

To expand the disturbing function $\mathcal{R}$, we need to express the unitary position vectors $\hat{\boldsymbol{r}}_{1}$ and $\hat{\boldsymbol{r}}_{2}$ as functions of the orbit elements of bodies 1 and 2 . According to orbital theory, the unitary vector $\hat{\boldsymbol{r}}_{1}$ can be written as

$\hat{\boldsymbol{r}}_{1}=\frac{\boldsymbol{r}_{1}}{r_{1}}=\left[\begin{array}{l}\cos \Omega_{1} \cos \theta_{1}-\sin \Omega_{1} \cos i_{1} \sin \theta_{1} \\ \sin \Omega_{1} \cos \theta_{1}+\cos \Omega_{1} \cos i_{1} \sin \theta_{1} \\ \sin i_{1} \sin \theta_{1}\end{array}\right]$,

where $\theta_{1}=\omega_{1}+f_{1}$ is the argument of lattitude. Then, considering the following relationship between the true and eccentric anomalies,

$\sin f_{1}=\frac{\sqrt{1-e_{1}^{2}} \sin E_{1}}{1-e_{1} \cos E_{1}}, \quad \cos f_{1}=\frac{\cos E_{1}-e_{1}}{1-e_{1} \cos E_{1}}$,

equation (9) can be expressed as an explicit function of the eccentric anomaly in the form:

$\hat{\boldsymbol{r}}_{1}=\frac{1}{1-e_{1} \cos E_{1}}\left[\begin{array}{l}\mathcal{A}_{1} \cos E_{1}+\mathcal{B}_{1} \sin E_{1}+\mathcal{C}_{1} \\ \mathcal{A}_{2} \cos E_{1}+\mathcal{B}_{2} \sin E_{1}+\mathcal{C}_{2} \\ \mathcal{A}_{3} \cos E_{1}+\mathcal{B}_{3} \sin E_{1}+\mathcal{C}_{3}\end{array}\right]$,

where

$\mathcal{A}_{1}=\cos \Omega_{1} \cos \omega_{1}-\cos i_{1} \sin \Omega_{1} \sin \omega_{1}, \quad \mathcal{B}_{1}=-\eta\left(\cos \Omega_{1} \sin \omega_{1}+\cos i_{1} \sin \Omega_{1} \cos \omega_{1}\right), \quad \mathcal{C}_{1}=-e_{1} \mathcal{A}_{1}$

$\mathcal{A}_{2}=\sin \Omega_{1} \cos \omega_{1}+\cos i_{1} \cos \Omega_{1} \sin \omega_{1}, \quad \mathcal{B}_{2}=-\eta\left(\sin \Omega_{1} \sin \omega_{1}-\cos i_{1} \cos \Omega_{1} \cos \omega_{1}\right), \quad \mathcal{C}_{2}=-e_{1} \mathcal{A}_{2}$

$\mathcal{A}_{3}=\sin i_{1} \sin \omega_{1}, \quad \mathcal{B}_{3}=\eta \sin i_{1} \cos \omega_{1}, \quad \mathcal{C}_{3}=-e_{1} \mathcal{A}_{3}$

with $\eta=\sqrt{1-e_{1}^{2}}$ as the usual eccentricity function.

In the coordinate system $o-x y z$, the unitary position vector $\hat{\boldsymbol{r}}_{2}$ associated with the disturbing body depends only on the true anomaly $f_{2}$ and is given by

$\hat{\boldsymbol{r}}_{2}=\frac{\boldsymbol{r}_{2}}{r_{2}}=\left[\begin{array}{c}\cos f_{2} \\ \sin f_{2} \\ 0\end{array}\right]$.

Replacing equations (10) and (11) in equation (2) yields

$\cos \psi=\frac{1}{1-e_{1} \cos E_{1}}\left[\Gamma_{1} \cos E_{1} \cos f_{2}+\Gamma_{2} \cos E_{1} \sin f_{2}+\Gamma_{3} \sin E_{1} \cos f_{2}+\Gamma_{4} \sin E_{1} \sin f_{2}+\Gamma_{5} \cos f_{2}+\Gamma_{6} \sin f_{2}\right]$,

where

$\Gamma_{1}=\mathcal{A}_{1}, \Gamma_{2}=\mathcal{A}_{2}, \Gamma_{3}=\mathcal{B}_{1}, \Gamma_{4}=\mathcal{B}_{2}, \Gamma_{5}=\mathcal{C}_{1}, \Gamma_{6}=\mathcal{C}_{2}$ 
Based on equation (12), the term $\cos ^{n-2 k} \psi$ appearing in the disturbing function can be expanded as

$$
\begin{aligned}
\cos ^{n-2 k} \psi= & \frac{1}{\left(1-e_{1} \cos E_{1}\right)^{n-2 k}} \sum_{j_{1}+j_{2}+j_{3}+j_{4}+j_{5}+j_{6}=n-2 k} \Lambda_{j_{1} j_{2} j_{3} j_{4} j_{5} j_{6}}^{n-2 \Gamma_{1}} \Gamma_{1}^{j_{1}} \Gamma_{2}^{j_{2}} \Gamma_{3}^{j_{3}} \Gamma_{4}^{j_{4}} \Gamma_{5}^{j_{5}} \Gamma_{6}^{j_{6}} \\
& \times\left(\cos E_{1}\right)^{j_{1}+j_{2}}\left(\sin E_{1}\right)^{j_{3}+j_{4}}\left(\cos f_{2}\right)^{j_{1}+j_{3}+j_{5}}\left(\sin f_{2}\right)^{j_{2}+j_{4}+j_{6}},
\end{aligned}
$$

where $j_{1}, j_{2}, j_{3}, j_{4}, j_{5}, j_{6} \in \mathbb{N}$ and the coefficients are calculated by the following recurrence relation:

$\Lambda_{j_{1} j_{2} j_{3} j_{4} j_{5} j_{6}}^{k}=\Lambda_{\left(j_{1}-1\right) j_{2} j_{3} j_{4} j_{5} j_{6}}^{k-1}+\Lambda_{j_{1}\left(j_{2}-1\right) j_{3} j_{4} j_{5} j_{6}}^{k-1}+\Lambda_{j_{1} j_{2}\left(j_{3}-1\right) j_{4} j_{5} j_{6}}^{k-1}+\Lambda_{j_{1} j_{2} j_{3}\left(j_{4}-1\right) j_{5} j_{6}}^{k-1}+\Lambda_{j_{1} j_{2} j_{3} j_{4}\left(j_{5}-1\right) j_{6}}^{k-1}+\Lambda_{j_{1} j_{2} j_{3} j_{4} j_{5}\left(j_{6}-1\right)}^{k-1}$,

starting with

$\Lambda_{000000}^{0}=1, \quad \Lambda_{100000}^{1}=1, \quad \Lambda_{010000}^{1}=1, \quad \Lambda_{001000}^{1}=1, \quad \Lambda_{000100}^{1}=1, \quad \Lambda_{000010}^{1}=1, \quad \Lambda_{000001}^{1}=1$.

Consequently, the disturbing potential, truncated at order $N$, can be arranged as

$$
\begin{aligned}
\mathcal{R}= & \frac{\mathcal{G} m_{2}}{a_{2}} \sum_{n \geq 2}^{N} \alpha^{n} \sum_{k \geq 0}^{\left[\frac{n}{2}\right]} \frac{(-1)^{k}}{2^{n}} \frac{(2 n-2 k) !}{k !(n-k) !(n-2 k) !} \sum_{j_{1}+j_{2}+j_{3}+j_{4}+j_{5}+j_{6}=n-2 k} \Lambda_{j_{1} j_{2} j_{3} j_{4} j_{5} j_{6}}^{n-2 k} \Gamma_{1}^{j_{1}} \Gamma_{2}^{j_{2}} \Gamma_{3}^{j_{3}} \Gamma_{4}^{j_{4}} \Gamma_{5}^{j_{5}} \Gamma_{6}^{j_{6}} \\
& \times \frac{1}{\left(1-e_{1} \cos E_{1}\right)^{n-2 k}}\left(\frac{r_{1}}{a_{1}}\right)^{n}\left(\cos E_{1}\right)^{j_{1}+j_{2}}\left(\sin E_{1}\right)^{j_{3}+j_{4}}\left(\frac{a_{2}}{r_{2}}\right)^{n+1}\left(\cos f_{2}\right)^{j_{1}+j_{3}+j_{5}}\left(\sin f_{2}\right)^{j_{2}+j_{4}+j_{6}},
\end{aligned}
$$

where the part associated with the test particle's eccentric anomaly is denoted by

$S_{n, k, j_{1} j_{2} j_{3} j_{4}}^{\mathrm{I}}=\frac{1}{\left(1-e_{1} \cos E_{1}\right)^{n-2 k}}\left(\frac{r_{1}}{a_{1}}\right)^{n}\left(\cos E_{1}\right)^{j_{1}+j_{2}}\left(\sin E_{1}\right)^{j_{3}+j_{4}}$

and the part related to the disturbing body's true anomaly is denoted by

$S_{n, j_{1} j_{2} j_{3} j_{4} j_{5} j_{6}}^{\mathrm{II}}=\left(\frac{a_{2}}{r_{2}}\right)^{n+1}\left(\cos f_{2}\right)^{j_{1}+j_{3}+j_{5}}\left(\sin f_{2}\right)^{j_{2}+j_{4}+j_{6}}$.

By substituting equation (14) in equation (7) and considering the notations of equations (15) and (16), the Hamiltonian of the test particle, truncated at order $N$, can be obtained as

$$
\begin{aligned}
\mathcal{H}= & -\frac{\mu^{2}}{2 L^{2}}-\frac{\mathcal{G} m_{2}}{a_{2}} \sum_{n \geq 2}^{N} \alpha^{n} \sum_{k \geq 0}^{\left[\frac{n}{2}\right]} \frac{(-1)^{k}}{2^{n}} \frac{(2 n-2 k) !}{k !(n-k) !(n-2 k) !} \sum_{j_{1}+j_{2}+j_{3}+j_{4}+j_{5}+j_{6}=n-2 k} \Lambda_{j_{1} j_{2} j_{3} j_{4} j_{5} j_{6}}^{n-2 k} \\
& \times \Gamma_{1}^{j_{1}} \Gamma_{2}^{j_{2}} \Gamma_{3}^{j_{3}} \Gamma_{4}^{j_{4}} \Gamma_{5}^{j_{5}} \Gamma_{6}^{j_{6}} S_{n, k, j_{1} j_{2} j_{3} j_{4}}^{\mathrm{I}} S_{n, j_{1} j_{2} j_{3} j_{4} j_{5} j_{6}}^{\mathrm{II}} \cdot
\end{aligned}
$$

\section{SINGLE-AVERAGED HAMILTONIAN}

As we focus on the long-term dynamics of a triple system, the short-term effects in the Hamiltonian have to be filtered out by means of the averaging approach. The averaging process is known as the secular approximation. As the first term in the Hamiltonian shown by equation (17) is the Kepler energy of the test particle, which keeps the same expression after averaging, we only need to perform averaging for the disturbing function $\mathcal{R}$.

In the disturbing function, the short-term effects come from two parts: one is related to the instantaneous motion of the test particle, and the other one is caused by the motion of the disturbing body. For a hierarchical triple system, by definition, the mean motion of the test particle is much larger than that of the disturbing body. As a first step, it is necessary to average the disturbing function over the mean anomaly of the test particle $\left(l=M_{1}\right)$ as follows:

$\langle\mathcal{R}\rangle=\frac{1}{2 \pi} \int_{0}^{2 \pi} \mathcal{R} \mathrm{d} M_{1}$

To calculate equation (18), we only need to compute the averaged form for the term $S_{n, k, j_{1} j_{2} j_{3} j_{4}}^{\mathrm{I}}$, given by

$$
\left\langle S_{n, k, j_{1} j_{2} j_{3} j_{4}}^{\mathrm{I}}\right\rangle=\frac{1}{2 \pi} \int_{0}^{2 \pi} \frac{1}{\left(1-e_{1} \cos E_{1}\right)^{n-2 k}}\left(\frac{r_{1}}{a_{1}}\right)^{n}\left(\cos E_{1}\right)^{j_{1}+j_{2}}\left(\sin E_{1}\right)^{j_{3}+j_{4}} \mathrm{~d} M_{1} .
$$

Using the following relationships:

$\frac{r_{1}}{a_{1}}=1-e_{1} \cos E_{1}, \quad \frac{\mathrm{d} M_{1}}{\mathrm{~d} E_{1}}=1-e_{1} \cos E_{1}$,

equation (19) becomes

$\left\langle S_{n, k, j_{1} j_{2} j_{3} j_{4}}^{\mathrm{I}}\right\rangle=\frac{1}{2 \pi} \int_{0}^{2 \pi}\left(1-e_{1} \cos E_{1}\right)^{2 k+1}\left(\cos E_{1}\right)^{j_{1}+j_{2}}\left(\sin E_{1}\right)^{j_{3}+j_{4}} \mathrm{~d} E_{1}$, 
where the term $\left(1-e_{1} \cos E_{1}\right)^{2 k+1}$ can be expanded as a power series in $e_{1}$ and $\cos E_{1}$ of the form:

$\left(1-e_{1} \cos E_{1}\right)^{2 k+1}=\sum_{s_{1} \geq 0}^{2 k+1}\left(\begin{array}{c}2 k+1 \\ s_{1}\end{array}\right)\left(-e_{1}\right)^{s_{1}}\left(\cos E_{1}\right)^{s_{1}}, \quad s_{1} \in \mathbb{N}$

with the binomial coefficients defined by $\left(\begin{array}{c}n \\ m\end{array}\right)=\frac{n !}{m !(n-m) !}(n \geq m$ and $n, m \in \mathbb{N})$. Thus, we have

$\left\langle S_{n, k, j_{1} j_{2} j_{3} j_{4}}^{\mathrm{I}}\right\rangle=\sum_{s_{1} \geq 0}^{2 k+1}\left(\begin{array}{c}2 k+1 \\ s_{1}\end{array}\right)\left(-e_{1}\right)^{s_{1}} \frac{1}{2 \pi} \int_{0}^{2 \pi}\left(\cos E_{1}\right)^{j_{1}+j_{2}+s_{1}}\left(\sin E_{1}\right)^{j_{3}+j_{4}} \mathrm{~d} E_{1}$.

For convenience, let us denote the integral part of equation (22) by

$A_{p, q}=\frac{1}{2 \pi} \int_{0}^{2 \pi} \sin ^{p} E_{1} \cos ^{q} E_{1} \mathrm{~d} E_{1}, \quad p, q \in \mathbb{N}$.

When $p$ and $q$ are both even numbers, equation (23) is calculated by

$A_{p, q}=\sum_{u \geq 0}^{p / 2}\left(\begin{array}{c}p / 2 \\ u\end{array}\right)\left(\begin{array}{c}q+2 u \\ q / 2+u\end{array}\right) \frac{(-1)^{u}}{2^{q+2 u}}, \quad u \in \mathbb{N}$,

otherwise it is equal to zero.

Replacing equation (23) in equation (22) yields

$\left\langle S_{n, k, j_{1} j_{2} j_{3} j_{4}}^{\mathrm{I}}\right\rangle=\sum_{s_{1} \geq 0}^{2 k+1}\left(\begin{array}{c}2 k+1 \\ s_{1}\end{array}\right)\left(-e_{1}\right)^{s_{1}} A_{j_{3}+j_{4}, j_{1}+j_{2}+s_{1}}$.

Thus, the single-averaged expression of the disturbing function is

$$
\begin{aligned}
\langle\mathcal{R}\rangle= & \frac{\mathcal{G} m_{2}}{a_{2}} \sum_{n \geq 2}^{N} \alpha^{n} \sum_{k \geq 0}^{\left[\frac{n}{2}\right]} \frac{(-1)^{k}}{2^{n}} \frac{(2 n-2 k) !}{k !(n-k) !(n-2 k) !} \sum_{j_{1}+j_{2}+j_{3}+j_{4}+j_{5}+j_{6}=n-2 k} \Lambda_{j_{1} j_{2} j_{3} j_{4} j_{5} j_{6}}^{n-2 k} \\
& \times \Gamma_{1}^{j_{1}} \Gamma_{2}^{j_{2}} \Gamma_{3}^{j_{3}} \Gamma_{4}^{j_{4}} \Gamma_{5}^{j_{5}} \Gamma_{6}^{j_{6}}\left\langle S_{n, k, j_{1} j_{2} j_{3} j_{4}}^{\mathrm{I}}\right\rangle S_{n, j_{1} j_{2} j_{3} j_{4} j_{5} j_{6}}^{\mathrm{II}},
\end{aligned}
$$

and the single-averaged form of the Hamiltonian becomes

$\langle\mathcal{H}\rangle=-\frac{\mu^{2}}{2 L^{2}}-\langle\mathcal{R}\rangle$.

Obviously, the single-averaged Hamiltonian is independent on the fast variable $l\left(=M_{1}\right)$. Thus, in the singly averaged dynamical environment, the conjugate momentum $L$ is conserved, leading to the fact that the semi-major axis of the test particle keeps stationary. As a consequence, the differential equations governing the evolutions of $l$ and $L$ can be ignored. The equations of motion, describing the remaining variables, can be given by the following canonical relations:

$\begin{array}{ll}\frac{\mathrm{d} g}{\mathrm{~d} t}=\frac{\partial\langle\mathcal{H}\rangle}{\partial G}, & \frac{\mathrm{d} G}{\mathrm{~d} t}=-\frac{\partial\langle\mathcal{H}\rangle}{\partial g}, \\ \frac{\mathrm{d} h}{\mathrm{~d} t}=\frac{\partial\langle\mathcal{H}\rangle}{\partial H}, & \frac{\mathrm{d} H}{\mathrm{~d} t}=-\frac{\partial\langle\mathcal{H}\rangle}{\partial h},\end{array}$

which can be expressed by means of the partial derivatives of the disturbing function as follows:

$\begin{array}{ll}\frac{\mathrm{d} g}{\mathrm{~d} t}=-\frac{\partial\langle\mathcal{R}\rangle}{\partial G}, & \frac{\mathrm{d} G}{\mathrm{~d} t}=\frac{\partial\langle\mathcal{R}\rangle}{\partial g}, \\ \frac{\mathrm{d} h}{\mathrm{~d} t}=-\frac{\partial\langle\mathcal{R}\rangle}{\partial H}, & \frac{\mathrm{d} H}{\mathrm{~d} t}=\frac{\partial\langle\mathcal{R}\rangle}{\partial h} .\end{array}$

Using the relationship between the time $t$ and the true anomaly of the disturbing body $f_{2}$,

$\mathrm{d} t=\frac{\mathcal{P}_{2}}{2 \pi} \frac{\left(1-e_{2}^{2}\right)^{3 / 2}}{\left(1+e_{2} \cos f_{2}\right)^{2}} \mathrm{~d} f_{2}$,

with $\mathcal{P}_{2}$ as the orbital period of the disturbing body, equation (28) can be transformed into the differential equations with $f_{2}$ as the time-like independent variable:

$\begin{array}{ll}\frac{\mathrm{d} g}{\mathrm{~d} f_{2}}=-\frac{\partial\langle\tilde{\mathcal{R}}\rangle}{\partial G}, & \frac{\mathrm{d} G}{\mathrm{~d} f_{2}}=\frac{\partial\langle\tilde{\mathcal{R}}\rangle}{\partial g}, \\ \frac{\mathrm{d} h}{\mathrm{~d} f_{2}}=-\frac{\partial\langle\tilde{\mathcal{R}}\rangle}{\partial H}, & \frac{\mathrm{d} H}{\mathrm{~d} f_{2}}=\frac{\partial\langle\tilde{\mathcal{R}}\rangle}{\partial h},\end{array}$ 
where the term $\langle\tilde{\mathcal{R}}\rangle$ is given by

$$
\begin{aligned}
\langle\tilde{\mathcal{R}}\rangle= & \frac{\mathcal{G} m_{2}}{a_{2}} \sum_{n \geq 2}^{N} \alpha^{n} \sum_{k \geq 0}^{\left[\frac{n}{2}\right]} \frac{(-1)^{k}}{2^{n}} \frac{(2 n-2 k) !}{k !(n-k) !(n-2 k) !} \sum_{j_{1}+j_{2}+j_{3}+j_{4}+j_{5}+j_{6}=n-2 k} \Lambda_{j_{1} j_{2} j_{3} j_{4} j_{5} j_{6}}^{n-2 k} \\
& \times \Gamma_{1}^{j_{1}} \Gamma_{2}^{j_{2}} \Gamma_{3}^{j_{3}} \Gamma_{4}^{j_{4}} \Gamma_{5}^{j_{5}} \Gamma_{6}^{j_{6}}\left\langle S_{n, k, j_{1} j_{2} j_{3} j_{4}}^{\mathrm{I}}\right\rangle \tilde{S}_{n, j_{1} j_{2} j_{3} j_{4} j_{5} j_{6}}^{\mathrm{II}}
\end{aligned}
$$

with

$$
\tilde{S}_{n, j_{1} j_{2} j_{3} j_{4} j_{5} j_{6}}^{\mathrm{II}}=\frac{\mathcal{P}_{2}}{2 \pi} \frac{\left(1-e_{2}^{2}\right)^{3 / 2}}{\left(1+e_{2} \cos f_{2}\right)^{2}} S_{n, j_{1} j_{2} j_{3} j_{4} j_{5} j_{6}}^{\mathrm{II}} .
$$

By replacing equation (16) in equation (32) and using a binomial expansion similar to equation (21), equation (32) becomes

$$
\tilde{S}_{n, j_{1} j_{2} j_{3} j_{4} j_{5} j_{6}}^{\mathrm{II}}=\frac{\mathcal{P}_{2}}{2 \pi} \frac{1}{\left(1-e_{2}^{2}\right)^{n-1 / 2}} \sum_{s_{2} \geq 0}^{n-1}\left(\begin{array}{c}
n-1 \\
s_{2}
\end{array}\right) e_{2}^{s_{2}}\left(\sin f_{2}\right)^{p}\left(\cos f_{2}\right)^{q},
$$

where $p=j_{2}+j_{4}+j_{6}$ and $q=j_{1}+j_{3}+j_{5}+s_{2}$. Substituting equation (33) in equation (31) leads to the following expression:

$$
\begin{aligned}
\langle\tilde{\mathcal{R}}\rangle= & \frac{\mathcal{P}_{2}}{2 \pi} \frac{\mathcal{G} m_{2}}{a_{2}} \sum_{n \geq 2}^{N} \alpha^{n} \sum_{k \geq 0}^{\left[\frac{n}{2}\right]} \sum_{j_{1}+j_{2}+j_{3}+j_{4}+j_{5}+j_{6}=n-2 k} \sum_{s_{1} \geq 0}^{2 k+1} \sum_{s_{2} \geq 0}^{n-1} \frac{(-1)^{k+s_{1}}}{2^{n}} \frac{(2 n-2 k) !}{k !(n-k) !(n-2 k) !} \Lambda_{j_{1} j_{2} j_{3} j_{4} j_{5} j_{6}}^{n-2 k}\left(\begin{array}{c}
2 k+1 \\
s_{1}
\end{array}\right)\left(\begin{array}{c}
n-1 \\
s_{2}
\end{array}\right) \\
& \times A_{j_{3}+j_{4}, j_{1}+j_{2}+s_{1}} \frac{e_{2}^{s_{2}}}{\left(1-e_{2}^{2}\right)^{n-1 / 2}}\left(e_{1}\right)^{s_{1}} \Gamma_{1}^{j_{1}} \Gamma_{2}^{j_{2}} \Gamma_{3}^{j_{3}} \Gamma_{4}^{j_{4}} \Gamma_{5}^{j_{5}} \Gamma_{6}^{j_{6}}\left(\sin f_{2}\right)^{p}\left(\cos f_{2}\right)^{q} .
\end{aligned}
$$

To rearrange equation (34), we use the following notation:

$\mathcal{F}=e_{1}^{s_{1}} \Gamma_{1}^{j_{1}} \Gamma_{2}^{j_{2}} \Gamma_{3}^{j_{3}} \Gamma_{4}^{j_{4}} \Gamma_{5}^{j_{5}} \Gamma_{6}^{j_{6}}$

and expand the trigonometric term in equation (34) as Fourier series:

$\sin ^{p} f_{2} \cos ^{q} f_{2}=\mathcal{C}_{0}^{p, q}+\sum_{l \geq 1}^{p+q}\left[\mathcal{C}_{l}^{p, q} \cos l f_{2}+\mathcal{S}_{l}^{p, q} \sin l f_{2}\right]$.

The coefficient $\mathcal{C}_{0}^{p, q}$ is a constant, representing the secular part of the trigonometric function, and the other terms stand for the periodic oscillations (short-term variations). The detailed expressions of the coefficients $\mathcal{C}_{l}^{p, q}$ and $\mathcal{S}_{l}^{p, q}$ are provided by equations (A1-A4) in Appendix A.

Using equations (35) and (36), equation (34) can be written as

$$
\begin{aligned}
\langle\tilde{\mathcal{R}}\rangle= & \frac{\mathcal{P}_{2}}{2 \pi} \frac{\mathcal{G} m_{2}}{a_{2}} \sum_{n \geq 2}^{N} \alpha^{n} \sum_{k \geq 0}^{\left[\frac{n}{2}\right]} \sum_{j_{1}+j_{2}+j_{3}+j_{4}+j_{5}+j_{6}=n-2 k} \sum_{s_{1} \geq 0}^{2 k+1} \sum_{s_{2} \geq 0}^{n-1} \frac{(-1)^{k+s_{1}}}{2^{n}} \frac{(2 n-2 k) !}{k !(n-k) !(n-2 k) !} \\
& \times \Lambda_{j_{1} j_{2} j_{3} j_{4} j_{5} j_{6}}^{n-2 k}\left(\begin{array}{c}
2 k+1 \\
s_{1}
\end{array}\right)\left(\begin{array}{c}
n-1 \\
s_{2}
\end{array}\right) \frac{e_{2}^{s_{2}} A_{j_{3}+j_{4}, j_{1}+j_{2}+s_{1}} \mathcal{F} \times\left[\mathcal{C}_{0}^{p, q}+\sum_{l \geq 1}^{n-2 k+s_{2}}\left[\mathcal{C}_{l}^{p, q} \cos l f_{2}+\mathcal{S}_{l}^{p, q} \sin l f_{2}\right]\right] .}{\left(1-e_{2}^{2}\right)^{n-1 / 2}} .
\end{aligned}
$$

Equation (37) can be decomposed into the long-term and periodic parts as follows:

$$
\langle\tilde{\mathcal{R}}\rangle=\langle\tilde{\mathcal{R}}\rangle_{\text {long-term }}+\langle\tilde{\mathcal{R}}\rangle_{\text {periodic }}
$$

where the long-term part is independent on the true anomaly $f_{2}$, while the periodic part is a function of $f_{2}$. Accordingly, the canonical variables can be divided into two parts:

$g=g_{\text {long-term }}+g_{\text {periodic }}=g^{*}+\delta g, \quad G=G_{\text {long-term }}+G_{\text {periodic }}=G^{*}+\delta G$,

$h=h_{\text {long-term }}+h_{\text {periodic }}=h^{*}+\delta h, \quad H=H_{\text {long-term }}+H_{\text {periodic }}=H^{*}+\delta H$.

The evolution of the long-term variables $\left(g^{*}, G^{*}, h^{*}, H^{*}\right)$ is governed by the equations of secular motion, which will be discussed in the subsequent section. Here, we are only interested in the evolution of the periodic variables $(\delta g, \delta G, \delta h, \delta H)$, which is described by the following differential equations:

$$
\begin{array}{ll}
\frac{\mathrm{d}(\delta g)}{\mathrm{d} f_{2}}=-\frac{\partial\langle\tilde{\mathcal{R}}\rangle_{\text {periodic }}}{\partial G}, & \frac{\mathrm{d}(\delta G)}{\mathrm{d} f_{2}}=\frac{\partial\langle\tilde{\mathcal{R}}\rangle_{\text {periodic }}}{\partial g}, \\
\frac{\mathrm{d}(\delta h)}{\mathrm{d} f_{2}}=-\frac{\partial\langle\tilde{\mathcal{R}}\rangle_{\text {periodic }}}{\partial H}, & \frac{\mathrm{d}(\delta H)}{\mathrm{d} f_{2}}=\frac{\partial\langle\tilde{\mathcal{R}}\rangle_{\text {periodic }}}{\partial h},
\end{array}
$$


where

$$
\begin{aligned}
\frac{\partial\langle\tilde{\mathcal{R}}\rangle_{\text {periodic }}}{\partial(g, h, G, H)}= & \frac{\mathcal{P}_{2}}{2 \pi} \frac{\mathcal{G} m_{2}}{a_{2}} \sum_{n \geq 2}^{N} \alpha^{n} \sum_{k \geq 0}^{\left[\frac{n}{2}\right]} \sum_{j_{1}+j_{2}+j_{3}+j_{4}+j_{5}+j_{6}=n-2 k} \sum_{s_{1} \geq 0}^{2 k+1} \sum_{s_{2} \geq 0}^{n-1} \frac{(-1)^{k+s_{1}}}{2^{n}} \frac{(2 n-2 k) !}{k !(n-k) !(n-2 k) !} \\
& \times \Lambda_{j_{1} j_{2} j_{3} j_{4} j_{5} j_{6}}^{n-2 k}\left(\begin{array}{c}
2 k+1 \\
s_{1}
\end{array}\right)\left(\begin{array}{c}
n-1 \\
s_{2}
\end{array}\right) \frac{e_{2}^{s_{2}} A_{j_{3}+j_{4}, j_{1}+j_{2}+s_{1}}}{\left(1-e_{2}^{2}\right)^{n-1 / 2}} \frac{\partial \mathcal{F}}{\partial(g, h, G, H)} \sum_{l \geq 1}^{n-2 k+s_{2}}\left[\mathcal{C}_{l}^{p, q} \cos l f_{2}+\mathcal{S}_{l}^{p, q} \sin l f_{2}\right] .
\end{aligned}
$$

In equation (40), the elements $(g, G, h, H)$ are slow variables. Thus, at any instant, we could approximate $(g, G, h, H)$ as constants equal to the long-term contributions, i.e. taking $(g, G, h, H)=\left(g^{*}, G^{*}, h^{*}, H^{*}\right)$. A similar assumption can be found in Ćuk \& Burns (2004). Under this assumption, it is observed that the functions on the right-hand side of equation (40) are periodic with respect to the true anomaly $f_{2}$. Consequently, the differential equations represented by equation (40) can be analytically solved, and the analytical solution can be written in the form of Fourier series. As an example, we provide the detailed expression for $\delta g$ as follows:

$$
\begin{aligned}
\delta g= & -\frac{\mathcal{P}_{2}}{2 \pi} \frac{\mathcal{G} m_{2}}{a_{2}} \sum_{n \geq 2}^{N} \alpha^{n} \sum_{k \geq 0}^{\left[\frac{n}{2}\right]} \sum_{j_{1}+j_{2}+j_{3}+j_{4}+j_{5}+j_{6}=n-2 k} \sum_{s_{1} \geq 0}^{2 k+1} \sum_{s_{2} \geq 0}^{n-1} \frac{(-1)^{k+s_{1}}}{2^{n}} \frac{(2 n-2 k) !}{k !(n-k) !(n-2 k) !} \Lambda_{j_{1} j_{2} j_{3} j_{4} j_{5} j_{6}}^{n-2 k} \\
& \times\left(\begin{array}{c}
2 k+1 \\
s_{1}
\end{array}\right)\left(\begin{array}{c}
n-1 \\
s_{2}
\end{array}\right) A_{j_{3}+j_{4}, j_{1}+j_{2}+s_{1}} \frac{\partial \mathcal{F}}{\partial G}\left\{\frac{e_{2}^{s_{2}}}{\left(1-e_{2}^{2}\right)^{n-1 / 2}} \sum_{l \geq 1}^{n-2 k+s_{2}}\left[\frac{1}{l} \mathcal{C}_{l}^{p, q} \sin l f_{2}-\frac{1}{l} \mathcal{S}_{l}^{p, q} \cos l f_{2}\right]\right\} .
\end{aligned}
$$

The explicit expressions for the other three periodic variables $(\delta G, \delta h, \delta H)$ can be obtained in a similar manner.

To summarize, the analytical expressions of all the four periodic variables can be rearranged in the following compact form:

$$
\begin{array}{ll}
\delta g=\sum_{l_{g} \geq 1}^{2 N-1}\left[\tilde{\mathcal{C}}_{l_{g}}^{g} \cos l_{g} f_{2}+\tilde{\mathcal{S}}_{l_{g}}^{g} \sin l_{g} f_{2}\right], & \delta G=\sum_{l_{G} \geq 1}^{2 N-1}\left[\tilde{\mathcal{C}}_{l_{G}}^{G} \cos l_{G} f_{2}+\tilde{\mathcal{S}}_{l_{G}}^{G} \sin l_{G} f_{2}\right], \\
\delta h=\sum_{l_{h} \geq 1}^{2 N-1}\left[\tilde{\mathcal{C}}_{l_{h}}^{h} \cos l_{h} f_{2}+\tilde{\mathcal{S}}_{l_{h}}^{h} \sin l_{h} f_{2}\right], & \delta H=\sum_{l_{H} \geq 1}^{2 N-1}\left[\tilde{\mathcal{C}}_{l_{H}}^{H} \cos l_{H} f_{2}+\tilde{\mathcal{S}}_{l_{H}}^{H} \sin l_{H} f_{2}\right],
\end{array}
$$

which, as a byproduct, provides a transformation approach between the single-averaged elements $(g, G, h, H)$ and the secular elements $\left(g^{*}, G^{*}, h^{*}, H^{*}\right)$. The expressions of the coefficients in equation (42) are given by equations (B1) and (B2) in Appendix B.

\section{DOUBLE-AVERAGED HAMILTONIAN WITH PERIODIC CORRECTION}

The single-averaged expression of the disturbing function, provided by equation (25), can be rewritten as

$$
\begin{aligned}
\langle\mathcal{R}\rangle= & \frac{\mathcal{G} m_{2}}{a_{2}} \sum_{n \geq 2}^{N} \alpha^{n} \sum_{k \geq 0}^{\left[\frac{n}{2}\right]} \frac{(2 n-2 k) !}{k !(n-k) !(n-2 k) !} \sum_{j_{1}+j_{2}+j_{3}+j_{4}+j_{5}+j_{6}=n-2 k} \sum_{s_{1} \geq 0}^{2 k+1} \frac{(-1)^{k+s_{1}}}{2^{n}}\left(\begin{array}{c}
2 k+1 \\
s_{1}
\end{array}\right) \\
& \times \Lambda_{j_{1} j_{2} j_{3} j_{4} j_{5} j_{6}}^{n-2 k} \mathcal{F} A_{j_{3}+j_{4}, j_{1}+j_{2}+s_{1}}\left(\frac{a_{2}}{r_{2}}\right)^{n+1}\left(\cos f_{2}\right)^{j_{1}+j_{3}+j_{5}}\left(\sin f_{2}\right)^{j_{2}+j_{4}+j_{6}},
\end{aligned}
$$

where $\mathcal{F}$ is defined in equation (35). Obviously, the disturbing potential is a function of the instantaneous position of the disturbing body. To eliminate the short-term effects in the Hamiltonian caused by the motion of the disturbing body, we need to perform a second average over its mean anomaly, defined by

$$
\langle\langle\mathcal{R}\rangle\rangle=\frac{1}{2 \pi} \int_{0}^{2 \pi}\langle\mathcal{R}\rangle \mathrm{d} M_{2}=\frac{\left(1-e_{2}^{2}\right)^{3 / 2}}{2 \pi} \int_{0}^{2 \pi} \frac{\langle\mathcal{R}\rangle}{\left(1+e_{2} \cos f_{2}\right)^{2}} \mathrm{~d} f_{2},
$$

where the relation $\mathrm{d} M_{2}=\frac{\left(1-e_{2}^{2}\right)^{3 / 2}}{\left(1+e_{2} \cos f_{2}\right)^{2}} \mathrm{~d} f_{2}$ has been used. Replacing equation (43) in equation (44), we have

$$
\begin{aligned}
\langle\langle\mathcal{R}\rangle\rangle= & \frac{1}{2 \pi} \frac{\mathcal{G} m_{2}}{a_{2}} \sum_{n \geq 2}^{N} \alpha^{n} \sum_{k \geq 0}^{\left[\frac{n}{2}\right]} \sum_{j_{1}+j_{2}+j_{3}+j_{4}+j_{5}+j_{6}=n-2 k} \sum_{s_{1} \geq 0}^{2 k+1} \sum_{s_{2} \geq 0}^{n-1} \frac{(-1)^{k+s_{1}}}{2^{n}} \frac{(2 n-2 k) !}{k !(n-k) !(n-2 k) !}\left(\begin{array}{c}
2 k+1 \\
s_{1}
\end{array}\right)\left(\begin{array}{c}
n-1 \\
s_{2}
\end{array}\right) \\
& \times \Lambda_{j_{1} j_{2} j_{3} j_{4} j_{5} j_{6}}^{n-2 k} \frac{e_{2}^{s_{2}}}{\left(1-e_{2}^{2}\right)^{n-1 / 2}} \int_{0}^{2 \pi} \mathcal{F} A_{j_{3}+j_{4}, j_{1}+j_{2}+s_{1}}\left(\cos f_{2}\right)^{q}\left(\sin f_{2}\right)^{p} \mathrm{~d} f_{2},
\end{aligned}
$$

where $p=j_{2}+j_{4}+j_{6}$ and $q=j_{1}+j_{3}+j_{5}+s_{2}$.

In the classical double-averaging approach, the elements $(g, G, h, H)$ of the test particle are assumed as constants equal to their long-term contributions $\left(g^{*}, G^{*}, h^{*}, H^{*}\right)$ during the second average, i.e. the periodic corrections are not taken into account. Nevertheless, the neglect of the periodic effects can lead to non-negligible errors when the perturbing body has a relatively strong influence upon the motion of the test particle, for instance in the case that the mass of the perturber is comparable (or larger) with respect to the total mass of the inner binary. 
Considering this point, the periodic oscillations of Delaunay's elements, given by equation (42), are embedded in the disturbing function during the second average. In this setting, the function $\mathcal{F}$ can be approximated at the first order as follows:

$\mathcal{F}=\mathcal{F}_{*}+\left.\frac{\partial \mathcal{F}}{\partial G}\right|_{*} \delta G+\left.\frac{\partial \mathcal{F}}{\partial H}\right|_{*} \delta H$

where $\mathcal{F}_{*}$ and $\left.\frac{\partial \mathcal{F}}{\partial(G, H)}\right|_{*}$ mean that the functions are evaluated at the long-term elements $\left(L^{*}, g^{*}, G^{*}, h^{*}, H^{*}\right)$. It is obvious that the last two terms in equation (46) stand for the periodic corrections to the function $\mathcal{F}$. In Ćuk \& Burns (2004), the authors assumed the disturbing body moving in a circular orbit, and they considered the 'evection' corrections of the argument of pericentre $\left(g=\omega_{1}\right)$ and longitude of the ascending node ( $h=\Omega_{1}$ ) at the quadrupole-level approximation, i.e. the periodic oscillations of $e_{1}$ and $i_{1}$ of the test particle (corresponding to $\delta G$ and $\delta H$ in this work) have been incorporated into the double averaging.

Substituting equation (46) in equation (45) leads to the double-averaged form of the disturbing function:

$$
\begin{aligned}
\langle\langle\mathcal{R}\rangle\rangle= & \frac{\mathcal{G} m_{2}}{a_{2}} \sum_{n \geq 2}^{N} \alpha^{n} \sum_{k \geq 0}^{\left[\frac{n}{2}\right]} \sum_{j_{1}+j_{2}+j_{3}+j_{4}+j_{5}+j_{6}=n-2 k} \sum_{s_{1} \geq 0}^{2 k+1} \sum_{s_{2} \geq 0}^{n-1} \frac{(-1)^{k+s_{1}}}{2^{n}} \\
& \times \frac{(2 n-2 k) !}{k !(n-k) !(n-2 k) !}\left(\begin{array}{c}
2 k+1 \\
s_{1}
\end{array}\right)\left(\begin{array}{c}
n-1 \\
s_{2}
\end{array}\right) \Lambda_{j_{1} j_{2} j_{3} j_{4} j_{5} j_{6}}^{n-2 k} \frac{e_{2}^{s_{2}} A_{j_{3}+j_{4}, j_{1}+j_{2}+s_{1}}}{\left(1-e_{2}^{2}\right)^{n-1 / 2}} \\
& \times \frac{1}{2 \pi} \int_{0}^{2 \pi}\left[\mathcal{F}_{*}+\left.\frac{\partial \mathcal{F}}{\partial G}\right|_{*} \delta G+\left.\frac{\partial \mathcal{F}}{\partial H}\right|_{*} \delta H\right]\left(\cos f_{2}\right)^{q}\left(\sin f_{2}\right)^{p} \mathrm{~d} f_{2} .
\end{aligned}
$$

If we set $\delta G=\delta H=0$, the classical expression for the double-averaged disturbing function is retrieved. If we set $e_{2}=0$ and truncate the disturbing function at order 2, equation (47) is reduced to the case discussed in Ćuk \& Burns (2004).

In equation (47), the first term in the integral part can be calculated by

$\mathcal{T}_{0}=\int_{0}^{2 \pi} \mathcal{F}_{*}\left(\cos f_{2}\right)^{q}\left(\sin f_{2}\right)^{p} \mathrm{~d} f_{2}=2 \pi \mathcal{F}_{*} A_{p, q}$,

and the second term is

$\mathcal{T}_{G}=\left.\int_{0}^{2 \pi} \frac{\partial \mathcal{F}}{\partial G}\right|_{*} \delta G\left(\cos f_{2}\right)^{q}\left(\sin f_{2}\right)^{p} \mathrm{~d} f_{2}=\left.\frac{\partial \mathcal{F}}{\partial G}\right|_{*} ^{2 N-1} \sum_{l_{G} \geq 1}^{2 \pi} \int_{0}\left[\tilde{\mathcal{C}}_{l_{G}}^{G} \cos l_{G} f_{2}+\tilde{\mathcal{S}}_{l_{G}}^{G} \sin l_{G} f_{2}\right]\left(\cos f_{2}\right)^{q}\left(\sin f_{2}\right)^{p} \mathrm{~d} f_{2}$.

For simplicity, let us define

$\mathcal{U}_{p, q}^{l_{c}}=\int_{0}^{2 \pi} \sin ^{p} f \cos ^{q} f \cos l_{c} f \mathrm{~d} f, \quad \mathcal{V}_{p, q}^{l_{s}}=\int_{0}^{2 \pi} \sin ^{p} f \cos ^{q} f \sin l_{s} f \mathrm{~d} f$

with $p, q, l_{c}, l_{s} \in \mathbb{N}$. The detailed expressions for computing equations (50) are provided by equations (C1-C4) in Appendix C.

Using the notations given in equation (50), equation (49) can be simplified as

$\mathcal{T}_{G}=\left.\frac{\partial \mathcal{F}}{\partial G}\right|_{*} ^{2 N-1}\left[\tilde{\mathcal{C}}_{l_{G} \geq 1}^{G} \mathcal{U}_{p, q}^{l_{G}}+\tilde{\mathcal{S}}_{l_{G}}^{G} \mathcal{V}_{p, q}^{\mathcal{l}_{G}}\right]$

Similarly, the third term in the integral part of equation (47) can be obtained as

$\mathcal{T}_{H}=\left.\int_{0}^{2 \pi} \frac{\partial \mathcal{F}}{\partial H}\right|_{*} \delta H\left(\cos f_{2}\right)^{q}\left(\sin f_{2}\right)^{p} \mathrm{~d} f_{2}=\left.\frac{\partial \mathcal{F}}{\partial H}\right|_{*} ^{2 N-1}\left[\tilde{\mathcal{C}}_{l_{H} \geq 1}^{H} \mathcal{U}_{p, q}^{l_{H}}+\tilde{\mathcal{S}}_{l_{H}}^{H} \mathcal{V}_{p, q}^{d_{H}}\right]$

Thus, the double-averaged disturbing function can be written as

$$
\begin{aligned}
\langle\langle\mathcal{R}\rangle\rangle= & \frac{\mathcal{G} m_{2}}{a_{2}} \sum_{n \geq 2}^{N} \alpha^{n} \sum_{k \geq 0}^{\left[\frac{n}{2}\right]} \sum_{j_{1}+j_{2}+j_{3}+j_{4}+j_{5}+j_{6}=n-2 k} \sum_{s_{1} \geq 0}^{2 k+1} \sum_{s_{2} \geq 0}^{n-1} \frac{(-1)^{k+s_{1}}}{2^{n}} \frac{(2 n-2 k) !}{k !(n-k) !(n-2 k) !}\left(\begin{array}{c}
2 k+1 \\
s_{1}
\end{array}\right)\left(\begin{array}{c}
n-1 \\
s_{2}
\end{array}\right) \\
& \times \Lambda_{j_{1} j_{2} j_{3} j_{4} j_{5} j_{6}}^{n-2 k} \frac{e_{2}^{s_{2}} A_{j_{3}+j_{4}, j_{1}+j_{2}+s_{1}}}{\left(1-e_{2}^{2}\right)^{n-1 / 2}} \times \frac{1}{2 \pi}\left[\mathcal{T}_{0}+\mathcal{T}_{G}+\mathcal{T}_{H}\right] .
\end{aligned}
$$

In particular, when $\mathcal{T}_{G}=\mathcal{T}_{H}=0$, equation (53) reduces to the classical form of the double-averaged disturbing potential.

In the doubly averaged environment, the Hamiltonian becomes

$\langle\langle\mathcal{H}\rangle\rangle=-\frac{\mu^{2}}{2 L^{*^{2}}}-\langle\langle\mathcal{R}\rangle\rangle$, 
and the equations of secular motion are given by the following canonical relations:

$$
\begin{aligned}
\frac{\mathrm{d} g^{*}}{\mathrm{~d} t}=\frac{\partial\langle\langle\mathcal{H}\rangle\rangle}{\partial G^{*}}, & \frac{\mathrm{d} G^{*}}{\mathrm{~d} t}=-\frac{\partial\langle\langle\mathcal{H}\rangle\rangle}{\partial g^{*}}, \\
\frac{\mathrm{d} h^{*}}{\mathrm{~d} t}=\frac{\partial\langle\langle\mathcal{H}\rangle\rangle}{\partial H^{*}}, & \frac{\mathrm{d} H^{*}}{\mathrm{~d} t}=-\frac{\partial\langle\langle\mathcal{H}\rangle\rangle}{\partial h^{*}} .
\end{aligned}
$$

The partial derivative of $\langle\langle\mathcal{H}\rangle\rangle$ with respect to $L^{*}$ produces the mean motion of the test particle (this differential equation is trivial and not presented here). About the explicit expressions of equation (55), please refer to equations (D1-D3) in Appendix D.

Equivalently, the equations of secular motion can be expressed in the form of Lagrange planetary equations:

$$
\begin{aligned}
\frac{\mathrm{d} e_{1}^{*}}{\mathrm{~d} t} & =\frac{\eta^{*}}{n_{1} a_{1}^{* 2} e_{1}^{*}} \frac{\partial\langle\langle\mathcal{H}\rangle\rangle}{\partial \omega_{1}^{*}}, \\
\frac{\mathrm{d} i_{1}^{*}}{\mathrm{~d} t} & =\frac{\csc i_{1}^{*}}{n_{1} a_{1}^{* 2} \eta^{*}}\left[\frac{\partial\langle\langle\mathcal{H}\rangle\rangle}{\partial \Omega_{1}^{*}}-\cos i_{1}^{*} \frac{\partial\langle\langle\mathcal{H}\rangle\rangle}{\partial \omega_{1}^{*}}\right], \\
\frac{\mathrm{d} \Omega_{1}^{*}}{\mathrm{~d} t} & =-\frac{\csc i_{1}^{*}}{n_{1} a_{1}^{* 2} \eta^{*}} \frac{\partial\langle\langle\mathcal{H}\rangle\rangle}{\partial i_{1}^{*}}, \\
\frac{\mathrm{d} \omega_{1}^{*}}{\mathrm{~d} t} & =\frac{\cot i_{1}^{*}}{n_{1} a_{1}^{* 2} \eta^{*}} \frac{\partial\langle\langle\mathcal{H}\rangle\rangle}{\partial i_{1}^{*}}-\frac{\eta^{*}}{n_{1} a_{1}^{* 2} e_{1}^{*}} \frac{\partial\langle\langle\mathcal{H}\rangle\rangle}{\partial e_{1}^{*}},
\end{aligned}
$$

where $\eta^{*}=\sqrt{1-e_{1}^{* 2}}, n_{1}$ is the mean motion determined by $n_{1}^{2} a_{1}^{* 3}=\mathcal{G} m_{0}$, and $\left(a_{1}^{*}, e_{1}^{*}, i_{1}^{*}, \Omega_{1}^{*}, \omega_{1}^{*}\right)$ are the secular (or mean) elements of the test particle. It is to be noted that $\langle\langle\mathcal{H}\rangle\rangle$ appearing in equation (56) has to be transformed into a function of the classical orbit elements by using the relationship given by equation (6).

In the following description, we will use the classical double-averaged Hamiltonian (or the classical approach) to stand for the model where the periodic corrections are neglected (i.e. setting $\mathcal{T}_{G}=\mathcal{T}_{H}=0$ ) and the modified double-averaged Hamiltonian (or the modified approach) to represent the model where the periodic corrections are considered.

\section{APPLICATIONS}

In the previous section, a modified version of the double-averaged Hamiltonian, shown by equation (54), has been formulated by considering the periodic corrections in the double averaging. Following this approach, it is possible to develop equations of secular motion up to an arbitrary order in the form of equation (55) or (56). In particular, if we neglect the periodic corrections in the double-averaged disturbing function, i.e. setting $\mathcal{T}_{G}=\mathcal{T}_{H}=0$ in equation (53), the secular Hamiltonian can be reduced to the classical form. Traditionally, the secular Hamiltonian truncated at the second order is called the quadrupole level of approximation, the secular Hamiltonian truncated at the third order is the octupole level of approximation, and the like. Certainly, our approach can reproduce the classical, low-order, double-averaged Hamiltonian by setting $N=2$ (or $N=3$ ) and neglecting the periodic corrections.

In this section, we will apply the modified secular Hamiltonian to a variety of hierarchical triple systems, covering stellar and planetary scales. It should be pointed out that our purpose is to validate (or test) the approach developed in this study and not to provide novel information about these systems.

\subsection{Planet's motion in a stellar binary system}

In this part, we apply the secular Hamiltonian to a stellar system consisting of a central star with mass $m_{0}=1 M_{\text {Sun }}$, a disturbing body with mass $m_{2}=1 M_{\text {Sun }}$ and a planet with mass $m_{1}$. The mass of the planet is infinitesimal compared to the other two massive bodies, so that the planet is approximated as a test particle $\left(m_{1} \rightarrow 0\right)$. Both the classical and the modified secular Hamiltonians are constructed up to order four (i.e. setting $N=4$ ), and their corresponding equations of secular motion are numerically integrated over $5 \times 10^{4} \mathrm{yr}$. The numerical integrator we used in this study is an eighth order Runge-Kutta algorithm with a seventh order for the automatic step-size control (Fehlberg 1968), and the absolute tolerance is set as $1.0 \times 10^{-12}$. Note that the initial conditions adopted in our numerical simulation are the same as the ones in Luo et al. (2016). Please refer to the caption of Fig. 2 for the full description.

The resulting evolutions of the orbit elements, including the eccentricity $\left(e_{1}\right)$, the inclination $\left(i_{1}\right)$, and the angular momentum along the $z$ direction $(H)$, are reported in Fig. 2, where the left-hand panels are for the results related to the classical double-averaging approach and the right-hand ones are for the results associated with the modified double-averaging approach. To compare the two averaged dynamical models (the classical and modified versions), the original non-averaged model (i.e. the three-body problem) is directly integrated over the same time period. The evolutions of the elements coming from the direct $N$-body integration are shown in black lines in Fig. 2 . It is worth noting that equation (42) is used to generate the initial conditions (in the form of osculating elements) for the numerical integration of the non-averaged model.

From the left-hand panels of Fig. 2, it is observed that, in the classical double-averaged system, the test particle's orbit inclination can periodically flip its orientation between prograde and retrograde (with respect to the outer orbit), and at the inversion epoch the eccentricity 

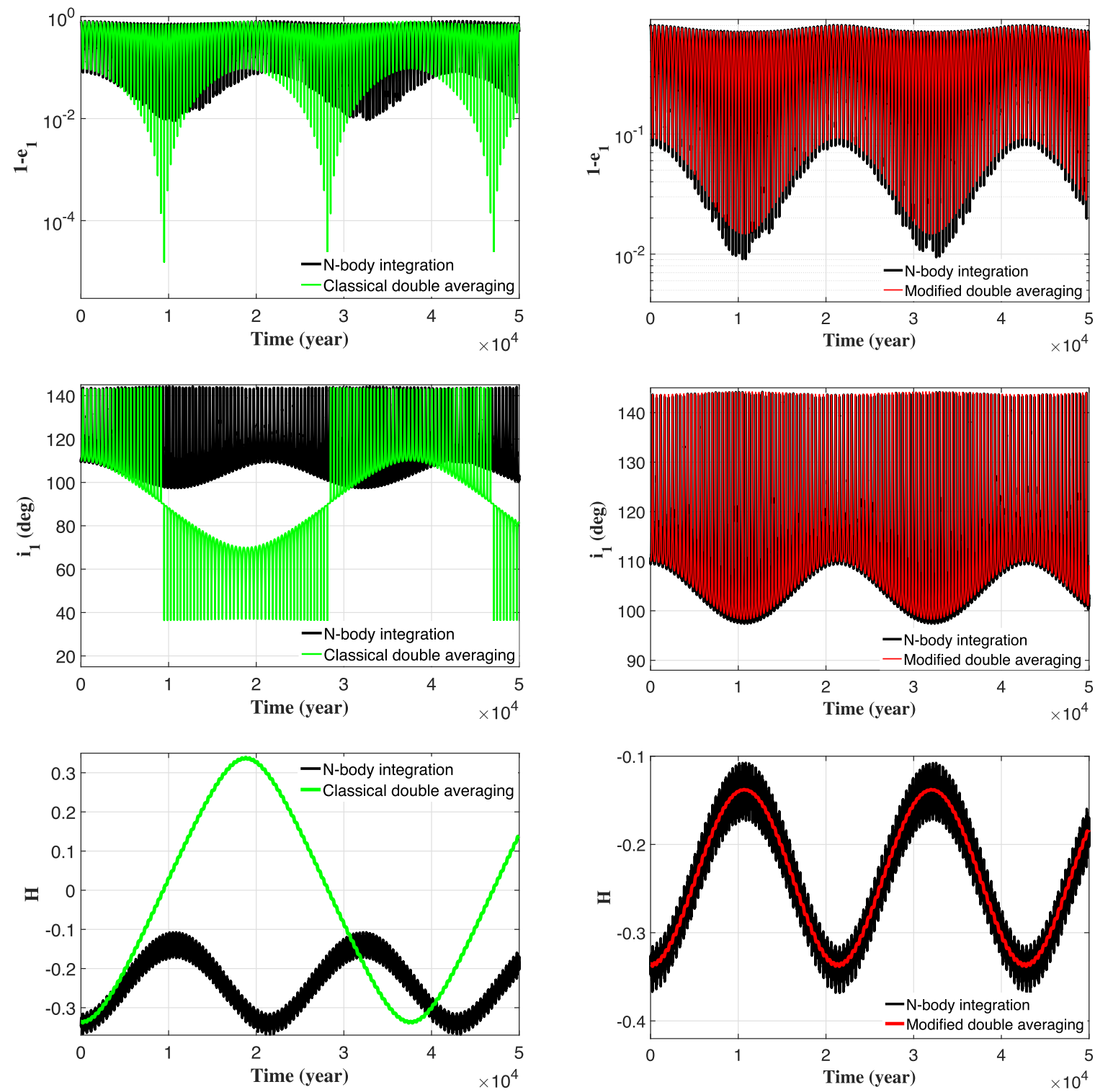

Figure 2. Time histories of the eccentricity excursion $\left(1-e_{1}\right)$, inclination $\left(i_{1}\right)$, and $z$ component of angular momentum $\left(H=G \cos i_{1}\right)$ of the test particle. In this triple system, the central body has the mass $m_{0}=1 M_{\text {Sun }}$, and the disturbing body has the mass $m_{2}=1 M_{\text {Sun. }}$. The initial condition of the test particle is assumed as $a_{1}=1 \mathrm{au}, e_{1}=0.2, i_{1}=110^{\circ}, \Omega_{1}=180^{\circ}$, and $\omega_{1}=0^{\circ}$, and the initial condition of the disturbing body is taken as $a_{2}=10$ au, $e_{2}=0.2$, and $i_{2}=\Omega_{2}=\omega_{2}=0^{\circ}$. The equations of secular motion truncated at the fourth order are integrated over $5 \times 10^{4} \mathrm{yr}$, including the dynamical model formulated by means of the classical double averaging technique (without considering periodic correction in the second average) and the one formulated by the modified double-averaging technique (considering periodic correction in the second average). The left three panels are for the classical double averaging, while the right panels are for the modified double averaging. The $\mathrm{N}$-body integration is also performed for the non-averaged three-body problem, and the time histories of osculating elements are given in black lines.

reaches an extremely high value which is close to unity. This process is usually referred to as the EKL mechanism (Naoz 2016). However, the actual evolution produced by the direct $N$-body integration shows that there are no occurrence of flipping for the considered initial conditions. The discrepancy implies that the classical secular Hamiltonian fails to produce the long-term behaviours for this case study. This is because the classical double-averaging approach ignores the periodic effects during the second average, which accumulate over a long time-scale and have significant contributions to the long-term behaviours, especially in the case that the perturbation coming from the disturbing body is relatively strong. Thus, neglecting these periodic effects in the classical double averaging may lead to dramatically different secular behaviours.

In contrast to the classical secular Hamiltonian, the modified version considers the accumulation of periodic oscillations during the second average. As expected, the resulting double-averaged system reproduces the long-term evolution in high precision, as shown in the right-hand panels of Fig. 2. Based on the simulation results, we observe: (a) the evolution of the inclination, predicted in the modified Hamiltonian, indicates that there are no flipping during the considered time period, which is in accordance with the conclusion derived from the $N$-body integration, (b) the evolution of $H=G \cos i_{1}$ obtained in the modified system is located in the middle of the variation produced by the $N$-body integration, and (c) the secular evolutions described by the modified Hamiltonian are in good agreement with the ones given 


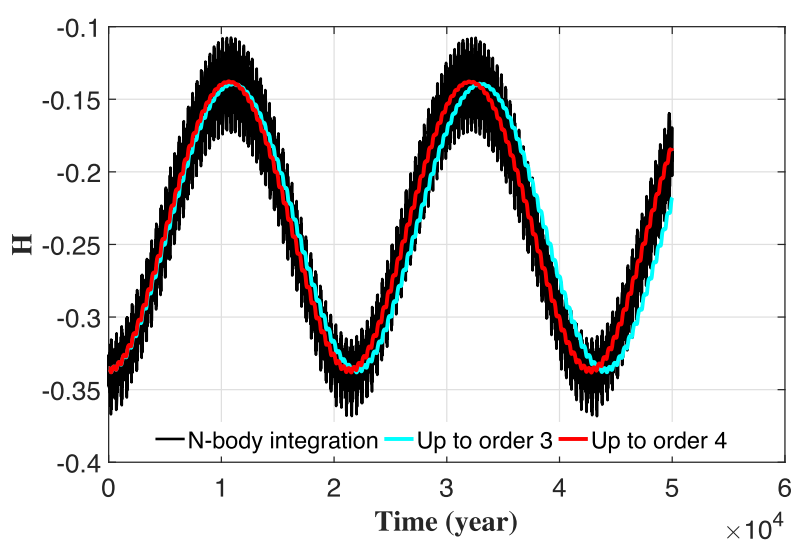

Figure 3. Time histories of the $z$ component of the angular momentum of the test particle $\left(H=G \cos i_{1}\right)$. The secular Hamiltonians are formulated by means of the modified double-averaging approach, and they are truncated at the third and fourth orders. The evolution of $H$ produced by means of the $N$-body integration is shown in black lines. The initial conditions are the same as the ones considered in Fig. 2.

by the direct $N$-body integration. These observations imply that the modified secular Hamiltonian formulated in this study is applicable in reproducing test particle's long-term behaviours.

Additionally, the secular Hamiltonian can be constructed up to different orders using the modified double-averaging approach developed in this study. A question arises: what is the advantage associated with a higher-order Hamiltonian? To answer this question, the equations of secular motion are constructed up to the third and fourth orders, and they are numerically integrated for a triple system with the same mass parameters and initial conditions as in Fig. 2. The time histories of the $z$ component of the angular momentum $(H)$ predicted by integrating the different-order double-averaged systems and the non-averaged three-body problem are reported in Fig. 3. As expected, the secular Hamiltonian truncated at a higher order has better accuracy in predicting long-term behaviours. About this point, Hamers \& Portegies (2016) have performed an analysis about the effects of high-order terms in the Hamiltonian upon secular evolutions for hierarchical multiple systems.

Note that the failure of the classical double-averaged Hamiltonian in predicting the long-term motion has been observed in Luo et al. (2016), who used a vectorial approach to address this problem in the octupole-level Hamiltonian by considering a quadrupole-level correction. In their study, the normalized angular momentum vector and the Runge-Lenz vector are used to describe the motions of test particles. For the same system and initial conditions (similar to the ones in Fig. 2), we compared our Hamiltonian approach with their vectorial method in the quadrupole-order Hamiltonian with the quadrupole-level corrections (by setting $N=2$ in our approach), concluding that our outcomes are identical to their results in the low-order setting.

\subsection{Satellites' motion in the Sun-planet systems}

In this part, we apply the double-averaged Hamiltonian to three Sun-planet-satellite systems, where the satellites are considered as test particles and the Sun acts as a perturber. These three systems are the Sun-perturbed Earth-Moon, Jupiter-Themisto, and Uranus-Caliban systems, where the planets are assumed to move around the Sun in fixed elliptic orbits. The semi-major axial ratios are 0.0026 for the Earth-Moon system, 0.0097 for the Jupiter-Themisto system and 0.0025 for the Uranus-Caliban system. Obviously, the ratios of semi-major axes of these systems are very small, thus in the practical computation it is accurate enough to construct the double-averaged Hamiltonians up to the third order (i.e. setting $N=3$ ). In fact, we have checked that introducing higher order terms $(N \geq 4)$ leads to negligible modifications compared to the present results.

For the Sun-perturbed Earth-Moon system, the evolutions of the Moon's orbit elements are reported in Fig. 4, where the black lines represent the results produced by the direct three-body integration, the green lines are the results related to the classical double averaging and the red lines are associated with the modified double averaging. For the description of the initial conditions, please refer to the caption. In the direct three-body integration, the elements of the Moon have short-term variations, and it is observed that the eccentricity varies in the range $(0.038,0.095)$ and the inclination in the range $\left(4.95^{\circ}, 5.3^{\circ}\right)$. In the doubly averaged environment, these short-term oscillations are averaged out, thus the elements (eccentricity and inclination) only have long-term variations where the amplitudes are greatly reduced compared to the direct integration results.

As for the evolutions of the longitude of the ascending node $\left(\Omega_{1}\right)$ and longitude of pericentre $\left(\Omega_{1}+\omega_{1}\right)$, the direct three-body integration indicates there are no evident short-term oscillations. We can observe that the long-term behaviours predicted by the modified double-averaged Hamiltonian are in better agreement with the direct integration results compared to the outcomes of the classical averaged Hamiltonian.

It is known that there exist nodal regression and apsidal precession periods for the Moon. The observed nodal regression period is about $18.6 \mathrm{yr}$, and the observed apsidal precession period is about $8.9 \mathrm{yr}$. From our simulation, it is observed that the apsidal precession and nodal regression rates are nearly constant, and it is possible to read their approximate values from Fig. 4 . The direct three-body integration shows a nodal regression period of about $18.16 \mathrm{yr}$ and an apsidal precession period of about $8.63 \mathrm{yr}$. The differences between our direct integration 

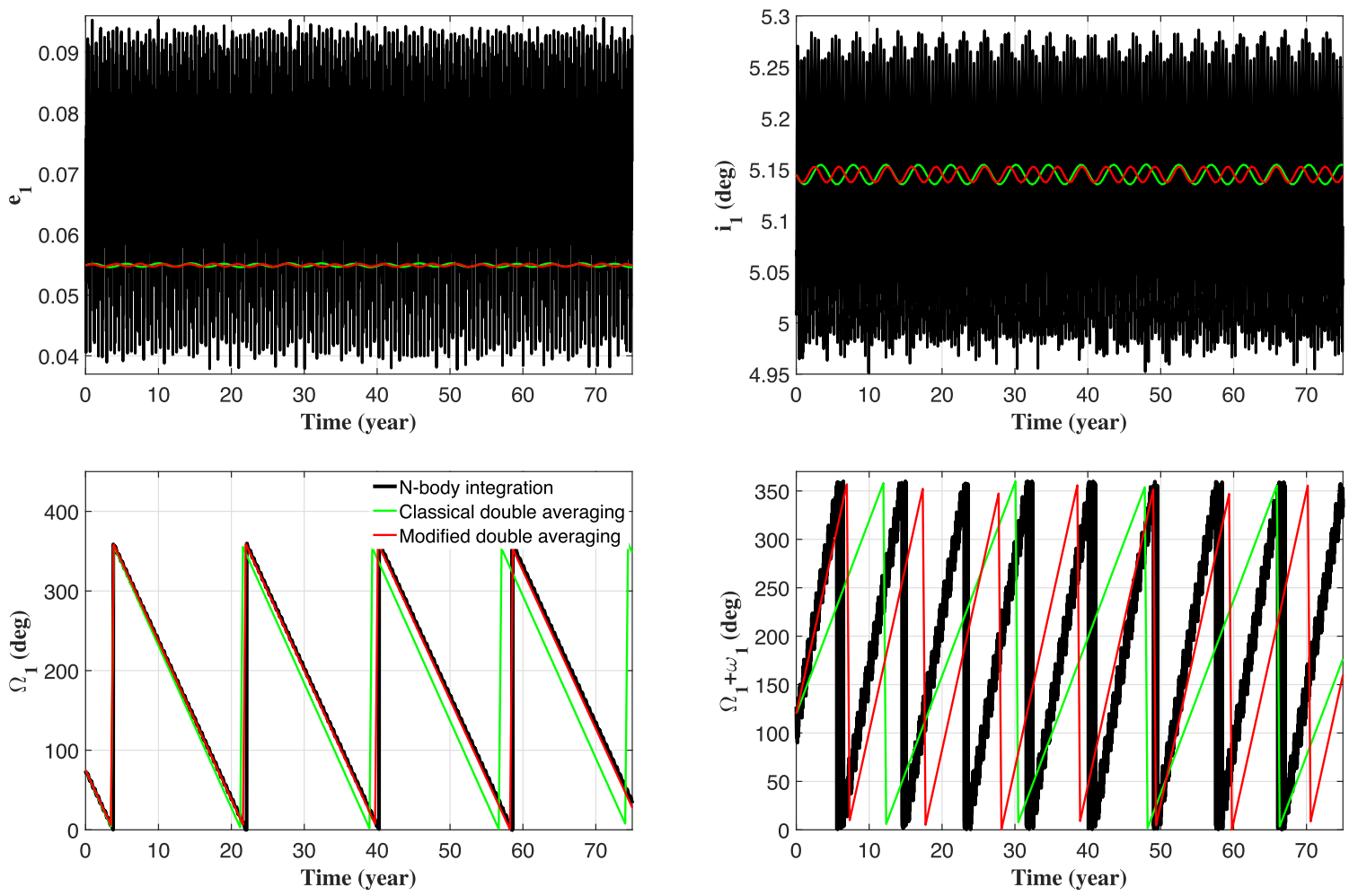

Figure 4. Evolutions of the orbit elements of the Moon including the eccentricity $\left(e_{1}\right)$, inclination $\left(i_{1}\right)$, longitude of the ascending node $\left(\Omega_{1}\right)$ and longitude of pericentre $\left(\Omega_{1}+\omega_{1}\right)$. The evolutions obtained by integrating the non-averaged three-body problem (the Sun-Earth-Moon system) are given in black lines, and the evolutions associated with the averaged models formulated by the classical and modified double-averaging approaches are marked in green and red lines, respectively. In our computation, the Moon is assumed as a massless body, and the Sun moves around the Earth in an elliptic orbit. The initial conditions of the Moon are assumed as $a_{1}=384748 \mathrm{~km}, e_{1}=0.0549, i_{1}=5.145^{\circ}, \Omega_{1}=75^{\circ}$, and $\omega_{1}=45^{\circ}$. The orbit of the Sun around the Earth is specified by $a_{2}=1$ au, $e_{2}=0.016$, and $i_{2}=\Omega_{2}=\omega_{2}=0^{\circ}$.

results and the observed periods are due to the approximations of our model: the test particle limit and the neglect of other perturbing bodies in the Solar system. The classical double-averaged model shows that the nodal regression and apsidal precession have the same periods (about $17.74 \mathrm{yr}$ ), indicating that the nodal period has an error of $0.86 \mathrm{yr}$ and the apsidal period is about twice as large as the observed value. About this issue, Clairaut in 1847 pointed out that the large error in precession periods is caused by the evection terms that are ignored in the double averaging, and this problem is often called 'lunar evection' (Baum \& Sheehan 2013).

In the modified double-averaged approach, the periodic effects (not only the evection terms) in the time-scale of the Sun's period are taken into account. The resulting double-averaged model produces a nodal regression period of about $18.13 \mathrm{yr}$ and an apsidal period of about 10.8 yr. Evidently, the consideration of short-term effects in the double averaging leads to a large correction to the apsidal period (from $17.74 \mathrm{yr}$ to $10.8 \mathrm{yr}$ ) and a relatively mall correction to the nodal period (from $17.74 \mathrm{yr}$ to $18.13 \mathrm{yr}$ ), making the predicted periods be closer to their observed values. The leaving errors are due to other effects neglected in our approach, such as the short-term effects in the time-scale of the lunar period (in our approach we only consider the periodic corrections in the time-scale of the solar period around the Earth), the gravitational attraction of the Moon to the Earth, orbit variations of the Sun around the Earth (actually it is a quasi-periodic orbit) and gravitational perturbations coming from other planets in the Solar system.

About the other two Sun-perturbed planet-satellite systems, the orbit variations obtained by integrating the non-averaged three-body problems and the double-averaged models formulated by the classical and modified double-averaging approaches are reported in Fig. 5 for Jupiter's satellite Themisto and in Fig. 6 for Uranus's satellite Caliban. The initial conditions for our numerical integrations are given in the the captions. From the simulation results, it is evident that the modified double-averaged Hamiltonian can produce more accurate long-term evolutions of elements than the classical version.

\subsection{Remarks}

As shown in the previous subsections, the modified double-averaged Hamiltonian developed in this work performs better than the classical version in reproducing the secular behaviour. However, some questions arise: is the modified approach always preferable? Is the modified Hamiltonian available for all hierarchical triple systems? To answer these questions, it is necessary to analyse the application conditions.

(i) In the classical double averaging, the elements of the test particle are taken as constants in the averages over the inner and outer binaries' orbital periods. Thus, the application conditions require that the elements of the test particle are slow variables in the time-scales of both the 

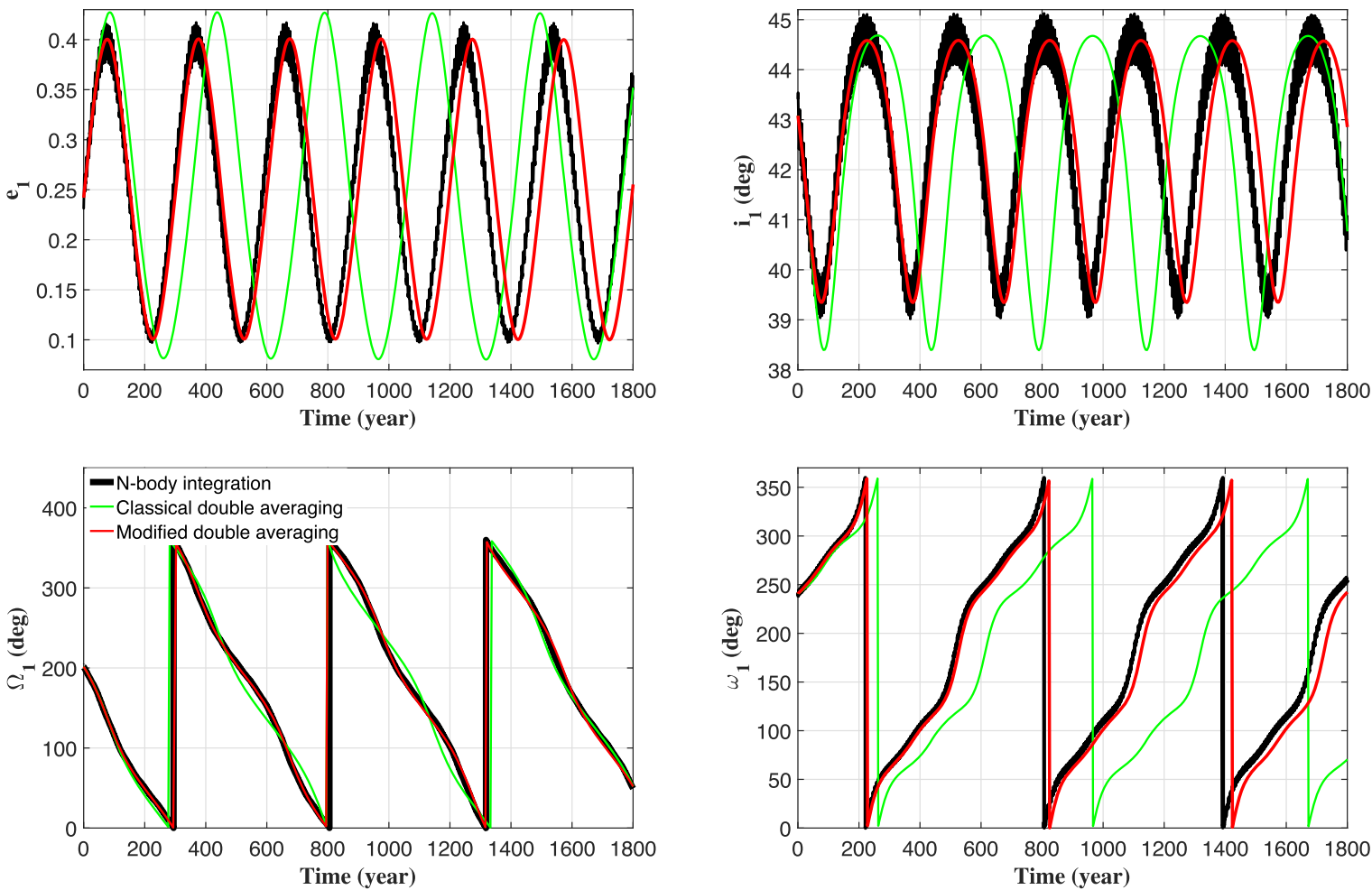

Figure 5. Similar to Fig. 4, but for Jupiter's satellite Themisto. The initial conditions of Themisto are set as $a_{1}=7507000 \mathrm{~km}, e_{1}=0.242, i_{1}=43.08^{\circ}$, $\Omega_{1}=201.5^{\circ}$, and $\omega_{1}=240.7^{\circ}$, and the orbit of the Sun moving around Jupiter is characterized by $a_{2}=5.2$ au, $e_{2}=0.049$, and $i_{2}=\Omega_{2}=\omega_{2}=0^{\circ}$.
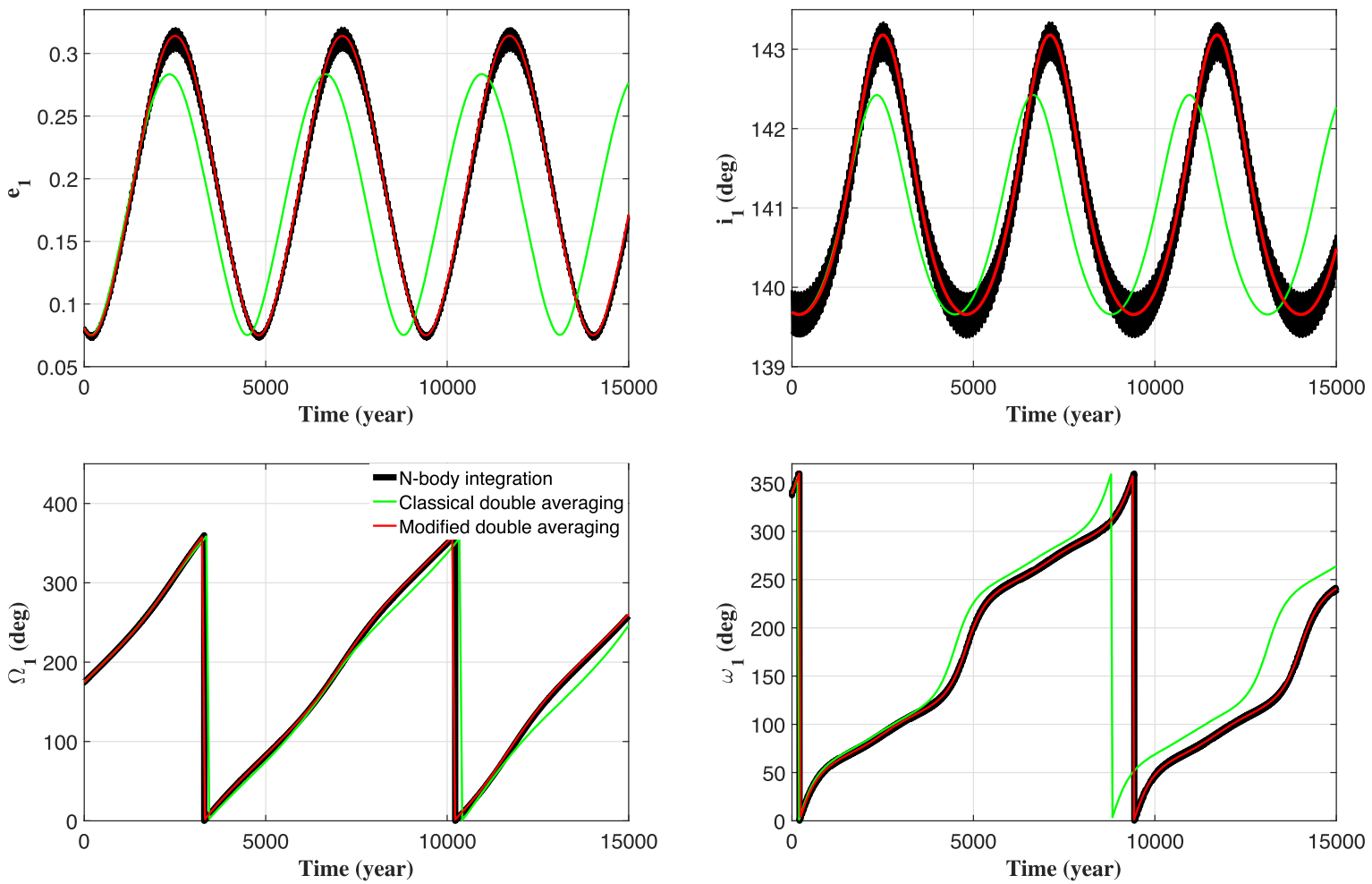

Figure 6. Similar to Fig. 4, but for Uranus's satellite Caliban. The initial conditions of Caliban are set as $a_{1}=0.047081 \mathrm{au}, e_{1}=0.0807123, i_{1}=139.68829^{\circ}$, $\Omega_{1}=174.99098^{\circ}$ and $\omega_{1}=339.07160^{\circ}$, and the orbit of the Sun moving around Uranus is characterized by $a_{2}=19.2184$ au, $e_{2}=0.044405586$, and $i_{2}=\Omega_{2}=\omega_{2}=0^{\circ}$. The elements of Caliban adopted here come from Yokoyama et al. (2003) (refer to fig. 1 in their work). 
Table 1. Computation time for the numerical integration of different-order averaged models about the Sun-perturbed UranusCaliban system. The initial conditions and time span are the same as the ones of Fig. 6 . The computation time about the full model over the same period is $9.720 \mathrm{~s}$.

\begin{tabular}{lcc}
\hline Order & $\begin{array}{c}\text { Time for the classical version } \\
(\mathrm{s})\end{array}$ & $\begin{array}{c}\text { Time for the modified version } \\
(\mathrm{s})\end{array}$ \\
\hline 2 & 1.265 & 1.437 \\
3 & 4.906 & 6.328 \\
4 & 17.047 & 23.703 \\
5 & 50.156 & 76.250 \\
\hline
\end{tabular}

inner and outer binaries' orbital periods (i.e. the secular frequencies of the elements need to be much smaller than the mean motion of the inner and outer binaries).

(ii) In the modified approach, we assumed that the elements of the test particle are constants in the average over the inner binary's orbital period (the first average) and took the periodic effects into consideration in the average over the outer binary's period (the second average). This means that the short-term effects in the time-scale of the outer binary's period are incorporated into the double-averaged Hamiltonian, but the short-term effects in the time-scale of the inner binary's period are neglected. Thus, the application conditions require that the elements of the test particle are slow variables in the time-scale of the inner binary's orbital period (i.e. the secular frequencies of the elements need to be much smaller than the mean motion of the inner binary).

It is evident that the modified approach broadens the application range compared to the classical double averaging. As for the computational efforts about the classical and modified double-averaged models, we report in Table 1 the time required by the numerical integration of different-order models over a time span of $1.5 \times 10^{4} \mathrm{yr}$ for the Sun-perturbed Uranus-Caliban system (the initial conditions and time span are the same as the ones of Fig. 6). It is observed that, compared to the classical approach, the modified version requires additional computation time. Therefore, when the application conditions of the classical approach are satisfied, the classical averaged model is preferable because in this case the periodic corrections considered in the modified approach become negligible but the computation time increases.

In some physical systems, such as Jupiter's irregular satellite systems, the strong perturbation coming from the disturbing body (the Sun) could make the elements of test particles no longer slow variables in the time-scale of the inner binary's orbital period. In this case, the short-term effects appearing in the first average could accumulate and produce significant errors to the secular evolution. To overcome this problem, the short-term effects would need to be incorporated also in the first average (over the inner binary's orbital period).

\section{CONCLUSIONS}

In this work, we developed a modified double-averaging approach, which was used to formulate a more accurate Hamiltonian in comparison to the secular Hamiltonian formulated by the classical double averaging. To test the modified approach, the resulting double-averaged Hamiltonian was applied to a variety of hierarchical triple systems, covering planetary, and stellar scales.

In this approach, the disturbing function appearing in the Hamiltonian is averaged twice in order to eliminate the short-term oscillations induced from the orbital motions of inner and outer binaries. When the perturbation coming from the disturbing body is relatively strong, the short-term effects neglected in the classical double averaging may accumulate over long time-scales. This leads to the consequence that the secular Hamiltonian formulated by the classical double-averaging approach produces different behaviours of the test particle in comparison with the results of the direct three-body integration. Based on such considerations, in this work we incorporated periodic corrections into the double averaging and derived a generalized version of the secular Hamiltonian, which can be constructed up to an arbitrary order. In particular, we provided two versions of secular evolution equations, given by the canonical relations and Lagrangian planetary equations.

To validate the modified double-averaging approach, for each example system we numerically integrated three dynamical models (the non-averaged three-body problem and the models formulated by means of the classical and modified double-averaging approaches) and compared their outcomes. First, the double-averaged Hamiltonian was applied to a stellar system, composed of a central star with mass $m_{0}=m_{\text {Sun }}$, a massless planet and a disturbing body with mass equal to that of the central star. The simulation results showed that the classical double-averaged Hamiltonian fails to predict the long-term behaviours, while the modified version reproduces the massless planet's long-term evolution in high accuracy. We compared the modified double-averaged Hamiltonians truncated at different orders, concluding that the outcomes produced in the secular system with inclusion of higher order terms have a better agreement with the evolution obtained by the direct three-body integration. Secondly, the double-averaged Hamiltonian was applied to three Sun-perturbed planet-satellite systems (the Earth-Moon system, Jupiter-Themisto system, and Uranus-Caliban system) to describe the secular motions of the satellites. We observed that, for all these systems, the modified double-averaged Hamiltonian has higher accuracy than the classical one in reproducing the secular behaviours.

We discussed the application conditions about the classical and modified double-averaged approaches. The classical approach requires that the secular frequencies of the test particle's elements are much smaller than the mean motion of the outer binary, while the modified approach requires that the secular frequencies of the elements are much smaller than the mean motion of the inner binary. Compared to the classical approach, the modified approach has a wider application range. In particular, for those physical systems where the disturbing body 
is comparable or more massive with respect to the total mass of the inner binary (in this case the classical double averaging may fail), the modified double-averaged Hamiltonian reproduces the secular behaviour with a high accuracy. However, when the application conditions of the classical and modified approaches are both satisfied, the classical approach is preferable due to the reduction of computation cost.

The modified double-averaged Hamiltonian could also be useful in predicting the secular behaviour of a spacecraft orbiting a planet or a moon in our Solar system. In fact, when the spacecraft moves in a high altitude orbit, the short-term effects induced by the perturbing body (the Sun for a planet's orbiter, the mother planet for a natural satellite's orbiter) can have significant influence upon its secular motion.

\section{ACKNOWLEDGEMENTS}

This work was accomplished during the first author's visit to Sapienza University of Rome. Lei H.L. thanks the financial support of the National Natural Science Foundation of China (No. 11603011, 41774038), the Natural Science Foundation of Jiangsu province (No. BK20160612), the visiting scholar program of China Scholarship Council (No. 201706195002), the National Basic Research Program 973 of China (2015CB857100), the National Defense Scientific Research Fund (No. 2016110C019) and the Fundamental Research Funds for the Central Universities (No. 020114380024).

\section{REFERENCES}

Antognini J. M. O., 2015, MNRAS, 452, 3610

Allan R. R., Cook G. E., 1964, Proc. R. Soc. Lond. Ser. A Math. Phys. Sci., 280, 97

Baum R., Sheehan W., 2013, Search of Planet Vulcan: The Ghost in Newton's Clockwork Universe. Springer-Verlag, Berlin

Carvalho J. P. S., Mourão D. C., de Moraes R. V., Prado A. F. B. A., Winter O. C., 2016, Celest. Mech. Dyn. Astr., 124, 73

Circi C., Condoleo E., Ortore E., 2017, Celest. Mech. Dyn. Astr., 128, 361

Condoleo E., Cinelli M., Ortore E., Circi C., 2016, J. Guid. Control Dyn., 2016, 2264

Ćuk M., Burns J. A., 2004, AJ, 128, 2518

Fehlberg E., 1968, Technical report NASA TR R-287, Classical fifth-, sixth-, seventh-, and eighth-order Runge-Kutta formulas with stepsize control. NASA Marshall Space Flight Center

Hamers A. S., Portegies Zwart S. F., 2016, MNRAS, 459, 2827

Harrington R. S., 1969, Celest. Mech., 1, 200

Katz B., Dong S., Malhotra R., 2011, Phys. Rev. Lett., 107, 181101

Kozai Y., 1962, AJ, 67, 591

Laskar J., Boué G., 2010, A\&A, 522, A60

Lei H. L., Xu B., 2013, MNRAS, 434, 1376

Lei H. L., Xu B., 2014, Commun. Non-Linear Sci. Numer. Simul., 19, 3374

Lei H. L., Xu B., Hou X., Sun Y., 2013, Celest. Mech. Dyn. Astr., 117, 349

Lei H. L., Xu B., Circi C., 2018, Celest. Mech. Dyn. Astr., 130, 38

Li G., Naoz S., Kocsis B., Loeb A., 2014a, ApJ, 785, 116

Li G., Naoz S., Holman M., Loeb A., 2014b, ApJ, 791, 86

Lidov M. L., 1962, P\&SS, 9, 719

Lithwick Y., Naoz S., 2011, ApJ, 742, 94

Luo L., Katz B., Dong S., 2016, MNRAS, 458, 3060

Naoz S., 2016, ARA\&A, 54, 441

Naoz S., Farr W. M., Lithwick Y., Rasio F. A., Teyssandier J., 2011, Nature, 473, 187

Naoz S., Farr W. M., Lithwick Y., Rasio F. A., Teyssandier J., 2013, MNRAS, 431, 2155

Naoz S., Li G., Zanardi M., de Elía G. C., Di Sisto R. P. et al., 2017, AJ, 154, 18

Petrovich C., 2015, ApJ, 805, 75

Thomas F., Morbidelli A., 1996, Celest. Mech. Dyn. Astr., 64, 209

Will C. M., 2017, Phys. Rev. D, 96, 023017

Yokoyama T., Santos M. T., Cardin G., Winter O. C., 2003, A\&A, 401, 763

\section{APPENDIX A: EXPANSIONS OF TRIGONOMETRIC FUNCTIONS}

The trigonometric function $\sin ^{p} f \cos ^{q} f(p, q \in \mathbb{N})$ can be expanded as Fourier series. In particular, when $p$ and $q$ are both odd, we have

$\sin ^{p} f \cos ^{q} f=\frac{1}{2^{p+q-1}} \sum_{u_{1} \geq 0}^{\frac{p-1}{2}} \sum_{u_{2} \geq 0}^{\frac{q-1}{2}}(-1)^{\frac{p-1}{2}-u_{1}}\left(\begin{array}{c}p \\ u_{1}\end{array}\right)\left(\begin{array}{c}q \\ u_{2}\end{array}\right) \times\left[\sin \left(p-2 u_{1}+q-2 u_{2}\right) f+\sin \left(p-2 u_{1}-q+2 u_{2}\right) f\right]$.

When $p$ is odd and $q$ is even, it is

$$
\begin{aligned}
\sin ^{p} f \cos ^{q} f= & \frac{1}{2^{p+q-1}}\left(\begin{array}{c}
q \\
q / 2
\end{array}\right) \sum_{u_{1} \geq 0}^{\frac{p-1}{2}}(-1)^{\frac{p-1}{2}-u_{1}}\left(\begin{array}{c}
p \\
u_{1}
\end{array}\right) \sin \left(p-2 u_{1}\right) f \\
& +\frac{1}{2^{p+q-1}} \sum_{u_{1} \geq 0}^{\frac{p-1}{2}} \sum_{u_{2} \geq 0}^{\frac{q}{2}-1}(-1)^{\frac{p-1}{2}-u_{1}}\left(\begin{array}{c}
p \\
u_{1}
\end{array}\right)\left(\begin{array}{c}
q \\
u_{2}
\end{array}\right) \times\left[\sin \left(p-2 u_{1}+q-2 u_{2}\right) f+\sin \left(p-2 u_{1}-q+2 u_{2}\right) f\right] .
\end{aligned}
$$


When $p$ is even and $q$ is odd, it is

$$
\begin{aligned}
\sin ^{p} f \cos ^{q} f= & \frac{1}{2^{p+q-1}}\left(\begin{array}{c}
p \\
p / 2
\end{array}\right) \sum_{u_{2} \geq 0}^{\frac{q-1}{2}}\left(\begin{array}{c}
q \\
u_{2}
\end{array}\right) \cos \left(q-2 u_{2}\right) f \\
& +\frac{1}{2^{p+q-1}} \sum_{u_{1} \geq 0}^{\frac{p}{2}-1} \sum_{u_{2} \geq 0}^{\frac{q-1}{2}}(-1)^{\frac{p}{2}-u_{1}}\left(\begin{array}{c}
p \\
u_{1}
\end{array}\right)\left(\begin{array}{c}
q \\
u_{2}
\end{array}\right)\left[\cos \left(p-2 u_{1}-q+2 u_{2}\right) f+\cos \left(p-2 u_{1}+q-2 u_{2}\right) f\right] .
\end{aligned}
$$

When $p$ and $q$ are both even, it is

$$
\begin{aligned}
\sin ^{p} f \cos ^{q} f= & \frac{1}{2^{p+q}}\left(\begin{array}{c}
p \\
p / 2
\end{array}\right)\left(\begin{array}{c}
q \\
q / 2
\end{array}\right)+\frac{1}{2^{p+q-1}}\left(\begin{array}{c}
p \\
p / 2
\end{array}\right) \sum_{u_{2} \geq 0}^{\frac{q}{2}-1}\left(\begin{array}{c}
q \\
u_{2}
\end{array}\right) \cos \left(q-2 u_{2}\right) f \\
& +\frac{1}{2^{p+q-1}}\left(\begin{array}{c}
q \\
q / 2
\end{array}\right) \sum_{u_{1} \geq 0}^{\frac{p}{2}-1}(-1)^{\frac{p}{2}-u_{1}}\left(\begin{array}{c}
p \\
u_{1}
\end{array}\right) \cos \left(p-2 u_{1}\right) f \\
& +\frac{1}{2^{p+q-1}} \sum_{u_{1} \geq 0}^{\frac{p}{2}-1} \sum_{u_{2} \geq 0}^{\frac{q}{2}-1}(-1)^{\frac{p}{2}-u_{1}}\left(\begin{array}{c}
p \\
u_{1}
\end{array}\right)\left(\begin{array}{c}
q \\
u_{2}
\end{array}\right)\left[\cos \left(p-2 u_{1}-q+2 u_{2}\right) f+\cos \left(p-2 u_{1}+q-2 u_{2}\right) f\right] .
\end{aligned}
$$

\section{APPENDIX B: COEFFICIENTS OF THE PERIODIC OSCILLATIONS}

The expressions of the coefficients in equation (42) are given by

$$
\begin{aligned}
\tilde{\mathcal{C}}_{l}^{(g, h)}= & -\frac{\mathcal{P}_{2}}{2 \pi} \frac{G m_{2}}{a_{2}} \sum_{n \geq 2}^{N} \alpha^{n} \sum_{k \geq 0}^{\left[\frac{n}{2}\right]} \sum_{j_{1}+j_{2}+j_{3}+j_{4}+j_{5}+j_{6}=n-2 k} \sum_{s_{1} \geq 0}^{2 k+1} \sum_{s_{2} \geq 0}^{n-1} \frac{(-1)^{k+s_{1}}}{2^{n}} \frac{(2 n-2 k) !}{k !(n-k) !(n-2 k) !} \Lambda_{j_{1} j_{2} j_{3} j_{4} j_{5} j_{6}}^{n-2 j_{6}} \\
& \times\left(\begin{array}{c}
2 k+1 \\
s_{1}
\end{array}\right)\left(\begin{array}{c}
n-1 \\
s_{2}
\end{array}\right) A_{j_{3}+j_{4}, j_{1}+j_{2}+s_{1}} \frac{\partial \mathcal{F}}{\partial(G, H)} \frac{e_{2}^{s_{2}}}{\left(1-e_{2}^{2}\right)^{n-1 / 2}} \sum_{t \geq 1, t-l=0}^{n-2 k+s_{2}}\left[-\frac{1}{t} \mathcal{S}_{t}^{p, q}\right], \\
\tilde{\mathcal{S}}_{l}^{(g, h)}= & -\frac{\mathcal{P}_{2}}{2 \pi} \frac{G m_{2}}{a_{2}} \sum_{n \geq 2}^{N} \alpha^{n} \sum_{k \geq 0}^{\left[\frac{n}{2}\right]} \sum_{j_{1}+j_{2}+j_{3}+j_{4}+j_{5}+j_{6}=n-2 k} \sum_{s_{1} \geq 0}^{2 k+1} \sum_{s_{2} \geq 0}^{n-1} \frac{(-1)^{k+s_{1}}}{2^{n}} \frac{(2 n-2 k) !}{k !(n-k) !(n-2 k) !} \Lambda_{j_{1} j_{2} j_{3} j_{4} j_{5} j_{6}}^{n-2 j_{6}} \\
& \times\left(\begin{array}{c}
2 k+1 \\
s_{1}
\end{array}\right)\left(\begin{array}{c}
n-1 \\
s_{2}
\end{array}\right) A_{j_{3}+j_{4}, j_{1}+j_{2}+s_{1}} \frac{\partial \mathcal{F}}{\partial(G, H)} \frac{e_{2}^{s_{2}}}{\left(1-e_{2}^{2}\right)^{n-1 / 2}} \sum_{t \geq 1, t-l=0}^{n-2 k+s_{2}}\left[\frac{1}{t} \mathcal{C}_{t}^{p, q}\right],
\end{aligned}
$$

and

$$
\begin{aligned}
\tilde{\mathcal{C}}_{l}^{(G, H)}= & \frac{\mathcal{P}_{2}}{2 \pi} \frac{G m_{2}}{a_{2}} \sum_{n \geq 2}^{N} \alpha^{n} \sum_{k \geq 0}^{\left[\frac{n}{2}\right]} \sum_{j_{1}+j_{2}+j_{3}+j_{4}+j_{5}+j_{6}=n-2 k} \sum_{s_{1} \geq 0}^{2 k+1} \sum_{s_{2} \geq 0}^{n-1} \frac{(-1)^{k+s_{1}}}{2^{n}} \frac{(2 n-2 k) !}{k !(n-k) !(n-2 k) !} \Lambda_{j_{1} j_{2} j_{3} j_{4} j_{5} j_{6}}^{n-2 k} \\
& \times\left(\begin{array}{c}
2 k+1 \\
s_{1}
\end{array}\right)\left(\begin{array}{c}
n-1 \\
s_{2}
\end{array}\right) A_{j_{3}+j_{4}, j_{1}+j_{2}+s_{1}} \frac{\partial \mathcal{F}}{\partial(g, h)} \frac{e_{2}^{s_{2}}}{\left(1-e_{2}^{2}\right)^{n-1 / 2}} \sum_{t \geq 1, t-l=0}^{n-2 k+s_{2}}\left[-\frac{1}{t} \mathcal{S}_{t}^{p, q}\right], \\
\tilde{\mathcal{S}}_{l}^{(G, H)}= & \frac{\mathcal{P}_{2}}{2 \pi} \frac{G m_{2}}{a_{2}} \sum_{n \geq 2}^{N} \alpha^{n} \sum_{k \geq 0}^{\left[\frac{n}{2}\right]} \sum_{j_{1}+j_{2}+j_{3}+j_{4}+j_{5}+j_{6}=n-2 k} \sum_{s_{1} \geq 0}^{2 k+1} \sum_{s_{2} \geq 0}^{n-1} \frac{(-1)^{k+s_{1}}}{2^{n}} \frac{(2 n-2 k) !}{k !(n-k) !(n-2 k) !} \Lambda_{j_{1} j_{2} j_{3} j_{4} j_{5} j_{6}}^{n-2 k} \\
& \times\left(\begin{array}{c}
2 k+1 \\
s_{1}
\end{array}\right)\left(\begin{array}{c}
n-1 \\
s_{2}
\end{array}\right) A_{j_{3}+j_{4}, j_{1}+j_{2}+s_{1}} \frac{\partial \mathcal{F}}{\partial(g, h)} \frac{e_{2}^{s_{2}}}{\left(1-e_{2}^{2}\right)^{n-1 / 2}} \sum_{t \geq 1, t-l=0}^{n-2 k+s_{2}}\left[\frac{1}{t} \mathcal{C}_{t}^{p, q}\right] .
\end{aligned}
$$

where $p=j_{2}+j_{4}+j_{6}$ and $q=j_{1}+j_{3}+j_{5}+s_{2}$. Concerning the notation used for summation $\sum_{t \geq 1, t-l=0}^{t_{\max }} f(t)$, the operation is performed when both the conditions $1 \leq t \leq t_{\max }$ and $t-l=0$ are satisfied (this operator also applies to Appendix D).

\section{APPENDIX C: DEFINITE INTEGRAL OF TRIGONOMETRIC FUNCTIONS}

The term $\mathcal{U}_{p, q}^{l_{c}}\left(p, q, l_{c} \in \mathbb{N}\right)$ is defined as

$\mathcal{U}_{p, q}^{l_{c}}=\int_{0}^{2 \pi} \sin ^{p} f \cos ^{q} f \cos l_{c} f \mathrm{~d} f$. 
If $p$ is even and $q, l_{c}$ are of the same parity, equation (C1) is calculated by

$$
\begin{aligned}
\mathcal{U}_{p, q}^{l_{c}}= & \int_{0}^{2 \pi} \frac{1}{2^{p}}\left(\begin{array}{c}
p \\
p / 2
\end{array}\right) \cos ^{q} f \cos \left(l_{c} f\right) \mathrm{d} f+\frac{2}{2^{p+1}} \sum_{u \geq 0}^{p / 2-1}(-1)^{p / 2-u}\left(\begin{array}{l}
p \\
u
\end{array}\right) \\
& \times \int_{0}^{2 \pi}\left[\cos ^{q} f \cos \left(p-2 u-l_{c}\right) f+\cos ^{q} f \cos \left(p-2 u+l_{c}\right) f\right] \mathrm{d} f,
\end{aligned}
$$

otherwise it equals zero.

The term $\mathcal{V}_{p, q}^{l_{s}}\left(p, q, l_{s} \in \mathbb{N}\right)$ is defined as

$\mathcal{V}_{p, q}^{l_{s}}=\int_{0}^{2 \pi} \sin ^{p} f \cos ^{q} f \sin l_{s} f \mathrm{~d} f$.

If $p$ is odd and $q, l_{s}$ are of the same parity, equation (C3) is determined by

$\mathcal{V}_{p, q}^{l_{s}}=\frac{2}{2^{p+1}} \sum_{u \geq 0}^{\frac{p-1}{2}}(-1)^{\frac{p-1}{2}-u}\left(\begin{array}{l}p \\ u\end{array}\right) \times \int_{0}^{2 \pi}\left[\cos ^{q} \lambda_{2} \cos \left(p-2 u-l_{s}\right) f-\cos ^{q} \lambda_{2} \cos \left(p-2 u+l_{s}\right) f\right] \mathrm{d} f$,

otherwise it is equal to zero.

The integral parts appearing in equations (C2) and (C4) are denoted by

$\mathcal{W}_{q}^{l_{c}}=\int_{0}^{2 \pi} \cos ^{q} f \cos l_{c} f \mathrm{~d} f,\left(q, l_{c} \in \mathbb{N}\right)$.

If $q$ and $l_{c}$ are of the same parity and $l_{c} \leq q$, equation (C5) is calculated by

$\mathcal{W}_{q}^{l_{c}}=\frac{\pi}{2^{q-1}}\left(\begin{array}{c}q \\ \frac{q-l_{c}}{2}\end{array}\right)$

otherwise it is equal to zero.

\section{APPENDIX D: EXPLICIT EXPRESSIONS OF THE DOUBLE-AVERAGED EQUATIONS OF MOTION}

To retrieve the explicit expressions of equation (55), it is required to provide the partial derivatives of the double-averaged disturbing function with respect to the Delaunay elements $\left(g^{*}, G^{*}, h^{*}, H^{*}\right)$. Therefore, according to equation (53), we need to derive the partial derivatives of $\mathcal{T}_{0}$, $\mathcal{T}_{G}$, and $\mathcal{T}_{H}$ relative to Delaunay elements.

The expression of $\mathcal{T}_{0}$ is given by equation (48), and its partial derivatives with respect to Delaunay elements can be retrieved by

$\frac{\partial \mathcal{T}_{0}}{\partial\left(g^{*}, G^{*}, h^{*}, H^{*}\right)}=2 \pi A_{p, q} \frac{\partial \mathcal{F}_{*}}{\partial\left(g^{*}, G^{*}, h^{*}, H^{*}\right)}$,

where the derivatives of $\mathcal{F}_{*}$ can be easily obtained from equation (35).

According to equation (51), the partial derivatives of $\mathcal{T}_{G}$ relative to Delaunay elements can be written as

$$
\begin{aligned}
\frac{\partial \mathcal{T}_{G}}{\partial\left(g^{*}, G^{*}, h^{*}, H^{*}\right)}= & \frac{\partial}{\partial\left(g^{*}, G^{*}, h^{*}, H^{*}\right)}\left[\left.\frac{\partial \mathcal{F}}{\partial G}\right|_{*}\right] \sum_{l_{G} \geq 1}^{2 N-1}\left[\widetilde{\mathcal{C}}_{l_{G}}^{G} \mathcal{U}_{p, q}^{l_{G}}+\widetilde{\mathcal{S}}_{l_{G}}^{G} \mathcal{V}_{p, q}^{l_{G}}\right] \\
& +\left.\frac{\partial \mathcal{F}}{\partial G}\right|_{*} ^{2 N-1}\left[\frac{\partial \widetilde{\mathcal{C}}_{l_{G}}^{G}}{\partial\left(g^{*}, G^{*}, h^{*}, H^{*}\right)} \mathcal{U}_{p, q}^{l_{G}}+\frac{\partial \widetilde{\mathcal{S}}_{l_{G}}^{G}}{\partial\left(g^{*}, G^{*}, h^{*}, H^{*}\right)} \mathcal{V}_{p, q}^{l_{G}}\right],
\end{aligned}
$$

where the first and second-order partial derivatives of $\mathcal{F}_{*}$ with respect to Delaunay elements can be easily obtained from equation (35). In equation (D2), the partial derivatives of $\widetilde{\mathcal{C}}_{l}^{G}$ and $\widetilde{\mathcal{S}}_{l}^{G}$ can be derived from equation (B2). They have the following form:

$$
\begin{aligned}
\frac{\partial \widetilde{\mathcal{C}}_{l_{G}}^{G}}{\partial\left(g^{*}, G^{*}, h^{*}, H^{*}\right)}= & \frac{P_{2}}{2 \pi} \frac{G m_{2}}{a_{2}} \sum_{n \geq 2}^{N} \alpha^{n} \sum_{k \geq 0}^{\left[\frac{n}{2}\right]} \sum_{j_{1}+j_{2}+j_{3}+j_{4}+j_{5}+j_{6}=n-2 k} \sum_{s_{1} \geq 0}^{2 k+1} \sum_{s_{2} \geq 0}^{n-1} \frac{(-1)^{k+s_{1}}}{2^{n}} \frac{(2 n-2 k) !}{k !(n-k) !(n-2 k) !} \Lambda_{j_{1} j_{2} j_{3} j_{4} j_{5} j_{6}}^{n-2 k} \\
& \times\left(\begin{array}{c}
2 k+1 \\
s_{1}
\end{array}\right)\left(\begin{array}{c}
n-1 \\
s_{2}
\end{array}\right) A_{j_{3}+j_{4}, j_{1}+j_{2}+s_{1}} \frac{\partial}{\partial\left(g^{*}, G^{*}, h^{*}, H^{*}\right)}\left[\left.\frac{\partial \mathcal{F}}{\partial g}\right|_{*}\right] \frac{e_{2}^{s_{2}}}{\left(1-e_{2}^{2}\right)^{n-1 / 2}} \sum_{l \geq 1, l-l_{G}=0}^{n-2 k+s_{2}}\left[-\frac{1}{l} \mathcal{S}_{l}^{p, q}\right], \\
\frac{\partial \widetilde{\mathcal{S}}_{l_{G}}^{G}}{\partial\left(g^{*}, G^{*}, h^{*}, H^{*}\right)}= & \frac{P_{2}}{2 \pi} \frac{G m_{2}}{a_{2}} \sum_{n \geq 2}^{N} \alpha^{n} \sum_{k \geq 0}^{\left[\frac{n}{2}\right]} \sum_{j_{1}+j_{2}+j_{3}+j_{4}+j_{5}+j_{6}=n-2 k}^{2 k+1} \sum_{s_{1} \geq 0}^{n-1} \frac{(-1)^{k+s_{1} \geq 0}}{2^{n}} \frac{(2 n-2 k) !}{k !(n-k) !(n-2 k) !} \Lambda_{j_{1} j_{2} j_{3} j_{4} j_{5} j_{6}}^{n-2 k} \\
& \times\left(\begin{array}{c}
2 k+1 \\
s_{1}
\end{array}\right)\left(\begin{array}{c}
n-1 \\
s_{2}
\end{array}\right) A_{j_{3}+j_{4}, j_{1}+j_{2}+s_{1}} \frac{\partial}{\partial\left(g^{*}, G^{*}, h^{*}, H^{*}\right)}\left[\left.\frac{\partial \mathcal{F}}{\partial g}\right|_{*}\right] \frac{e_{2}^{s_{2}}}{\left(1-e_{2}^{2}\right)^{n-1 / 2}} \sum_{l \geq 1, l-l_{G}=0}^{n-2 k+s_{2}}\left[\frac{1}{l} \mathcal{C}_{l}^{p, q}\right] .
\end{aligned}
$$


The partial derivatives of $\mathcal{T}_{H}$ relative to Delaunay elements can be derived in a similar manner, and their explicit expressions are similar to equation (D2).

By substituting equations (D1-D2) and the partial derivatives of $\mathcal{T}_{H}$ in the double-averaged Hamiltonian, it is possible to obtain the explicit expressions of equation (55).

This paper has been typeset from a $\mathrm{T}_{\mathrm{E}} \mathrm{X} / \mathrm{L} \mathrm{T}_{\mathrm{E}} \mathrm{X}$ file prepared by the author. 